\title{
Role of strain-dependent weakening memory on the style of mantle convection and plate boundary stability
}

\author{
Lukas Fuchs ${ }^{1,3^{*}}$ and Thorsten W. Becker ${ }^{1,2}$
}

${ }^{1}$ Institute for Geophysics, Jackson School of Geosciences, The University of Texas at Austin, Austin, Texas, USA

${ }^{2}$ Department of Geological Sciences, Jackson School of Geoscience, The University of Texas at Austin, Austin, Texas, USA

${ }^{3}$ now at Johann-Wolfgang Goethe Universität, Frankfurt, Germany

Manuscript submitted to Geophysical Journal International

* Corresponding author: J.J. Pickle Research Campus, Building 196, 10100 Burnet Road (R2200), Austin, TX 78758-4445, 512-987-3427, lfuchs@ig.utexas.edu 


\begin{abstract}
How plate tectonic surface motions relate to the convecting mantle remains one of the major problems in geosciences. In particular, the cause and consequence of strain localization at plate boundaries remains debated, even though strain memory, i.e. the ability to preserve and reactivate tectonic inheritance over geological time, appears to be a critical feature in plate tectonics. Here, we analyze how a parameterized damage weakening rheology, strain-dependent weakening, affects the time-dependence of plate boundary formation, the transition between mobile and stagnant-lid, and the reorganization of plates in 2-D convection models. The strain-dependent weakening within our models allows for a self-consistent formation and preservation of lithospheric weak zones, which are formed as remnants of subduction zones due to large-scale compressional deformation in the trench region. Such inherited weak zones can be reactivated as intra-plate subduction zones, ridge adjacent subduction, or as spreading centers themselves. Due to the weakening along plate boundaries, the inherited weak zones, and partly the accumulated strain along spreading centers, which weakens the shallow parts of the lithosphere, the longevity of mobile-lid convection increases. Strain-dependent weakening also enhances strain localization along convergent plate boundaries which increases their stability and longevity. As a consequence, tectonic inheritance is an important contribution to understanding the time-dependence of plate reorganization. Strain-dependent weakening results in a shift of the mobile-stagnant lid transition to higher effective yield stresses, if the weak zones fully penetrate the lithosphere and are relatively weakened by at least $20 \%$.
\end{abstract}

Keywords: Planetary tectonics; Dynamics of lithosphere and mantle; Kinematics of crustal and mantle deformation; Numerical modelling 


\section{Introduction}

How tectonic plate motions are generated by mantle convection on Earth and possibly other terrestrial-type planets is of fundamental importance for our understanding of planetary evolution and has been explored with a range of convection computations of increasing realism (e.g. Tackley, 2000a, b; van Heck \& Tackley, 2008; Foley \& Becker, 2009; Coltice et al., 2017). Earth's style of plate tectonics is characterized by a dichotomy between oceanic and continental plates due to fractionation, and in terms of kinematics by relatively rigid plates whose boundaries are defined as localizing all deformation in the strictest sense of the theory.

A key question is then how exactly Earth's tectonic surface motion is governed by the relatively strong and weakly to non-deforming plates surrounded by weak and strain-localizing plate boundaries. Besides the presence of continents, which affect surface motion in plate tectonics (e.g. Zhong, 2001; Rolf \& Tackley, 2011; Rolf et al. 2017), localization of strain and some sort of weakening along plate boundaries appears essential for plate-like surface motion (e.g. Bercovici, 1993; Zhong et al., 1998; Bercovici, 2003). Different mechanisms (e.g. plastic yielding, melt-reduced viscosities, variable grain size, shape preferred orientation of weak phases, lattice preferred orientation of olivine leading to mechanical anisotropy, dissipative heating, or damage weakening) have been suggested to create such localized, dynamically weakened boundaries (e.g. Schubert \& Turcotte, 1972; Tackley, 1998; Tommasi et al., 2009; Ricard \& Bercovici, 2009; Rozel et al., 2011; Montési, 2013), and some of these mechanisms have been explored in thermal convection models (e.g. Hall and Parmentier, 2003; Landuyt \& Bercovici 2009; Dannberg et al., 2017). However, to what extent over Earth's history strain localization and weakening along the plate boundaries are affected by which mechanism remains debated.

A primary aspect of Earth-like mantle convection is the temperature-dependent viscosity of mantle rocks (e.g. Solomatov, 1995; Zhong et al. 2000) leading to a cold, stiff, upper thermal boundary layer whose 
strength is reduced, for example, by plastic yielding (e.g. Kohlstedt et al. 1995) or a power-law rheology (e.g. Weinstein \& Olsen, 1992; Richards, 2001). A temperature-dependent viscosity and pseudo-plastic yielding (constant, and/or depth-dependent) lead to a roughly plate-like behavior (e.g. Tackley, 2000a), as quantified by the ratio of surface to interior motion (mobility), the degree of strain-rate focusing at plate boundaries (plateness), and the toroidal/poloidal ratio (Tackley, 2000b). Such behavior is also found in more recent spherical models which use stronger temperature dependence than earlier approaches (Coltice et al., 2017).

However, the yield stress found in all such spherical convection, "plate tectonics" generating models (e.g. van Heck \& Tackley, 2008; Foley \& Becker, 2009) tends to be smaller than the experimentally determined lithospheric strength of rocks. This may be because no true plastic yielding but rather some effectively smoothed version thereof is implemented, because other effects such as hydration might lower the effective yield stress of rocks, because Rayleigh numbers are still usually too low compared to Earth, or because of missing rheological mechanisms. Moreover, plastic yielding or power-law rheology alone do not lead to strain localization and formation of narrow, localized plate boundaries or pronounced transform faults (e.g. Bercovici, 1993; Tackley, 2000a; Bercovici et al., 2000; Landuyt et al. 2008; Gerya, 2010).

Rheological weakening, on the other hand, either due to velocity or strain rate weakening, can improve strain localization by increasing lubrication along plate boundaries. One such description is a grain-size dependent rheology with steady-state grain size, which leads to a strongly non-linear power law behavior. Crucially, any such instantaneous or 'steady-state' rheologies do not capture any memory effect of tectonics, such as inherited weak zones which can be reactivated. The memory of deformation and reactivation of weak zones in the lithosphere, however, appears to be a critical feature in plate tectonics (e.g. Wilson, 1966; Sykes, 1978; Gurnis et al., 2000). Thus, it remains debated how well, or how plate- 
tectonics like, the behavior and plate boundary reorganizations are captured in models which typically exclude the effects of deformation history and memory (but see e.g. Tackley, 2000b; Ogawa, 2003).

Besides instantaneous rheologies, strain localization and weakening has also been proposed and described by a more advanced description of damage mechanisms, controlled either by void or grain-size weakening (e.g. Bercovici and Ricard, 2005; Bercovici et al. 2015). While volatile or void weakening leads to strength reduction in the upper part of the lithosphere, the lower part of the lithosphere is probably not affected due to higher lithostatic pressures, which prevent full yielding (Landuyt \& Bercovici, 2009) and, in addition, provide only a minor plate-like behavior (Bercovici \& Ricard, 2005). Alternatively, the presence of small grain sizes in localized shear zones in mantle mylonites supports the idea of a grain-size dependent shearlocalizing feedback (e.g. Braun et al., 1999; Montési \& Hirth, 2003).

Grain-size dependent rheologies control the self-consistent dynamic weakening and effective inheritance in which grain-size reduction is controlled by dynamic recrystallization (e.g. Karato et al., 1980) and grainsize coarsening by normal grain growth (e.g. Karato, 1989; Austin \& Evans, 2007). However, it is unclear how effective grain-size weakening feedback mechanisms (e.g. Solomatov, 2001; Rozel et al, 2011; Hansen et al., 2012) as well as healing of weak zones due to grain growth, at least in single-phase systems (e.g. Karato et al. 1989; Braun et al., 1999; Ricard \& Bercovici, 2009; Rozel et al., 2011), could be for strain localization and tectonic inheritance (e.g. Bercovici et al., 2015). Moreover, grain-size evolution is less well constrained and including variable grain-size rheologies in global convection models remains computationally demanding (Dannberg et al., 2017).

Strain localization and weakening in the lithosphere has been observed and inferred to in nature (e.g. Audet \& Bürgmann, 2011; Précigout \& Almqvist, 2014) as well as described in models by many different processes, including non-Newtonian plastic rheology and yielding (e.g. Richards et al., 2001), velocity or 
pseudostick-slip rheology (e.g. Bercovici 1993, 1995), thermal weakening (e.g. Schubert \& Turcotte, 1972; Thielman \& Kaus, 2012), water and void weakening (e.g. Bercovici and Ricard, 2005; Landuyt \& Bercovici, 2009), dynamic recrystallization and grain-size evolution (e.g. Karato et al., 1980; Solomatov, 2001; Ricard and Bercovici, 2009; Rozel et al., 2011; Bercovici \& Ricard, 2012), or reactivation of preexisting/prescribed weak zones (e.g. Zhong \& Gurnis, 1995b; Bercovici \& Ricard, 2014; Mazzotti \& Gueydan, 2018).

Besides in mantle convection models, strain localization and weakening and such rheologies have been used widely in visco-elasto-plastic lithospheric deformation models (e.g. Lavier et al., 2000; Huismans \& Beaumont, 2003; Gerya, 2010; Gueydan et al. 2014). For example, strain weakening and localization have a significant effect on the symmetry of continental rift systems and general lithospheric break up. Moreover, the presence of inherited lithospheric weak zones can initiate or facilitate rifting, as rift systems do frequently form along zones of tectonic inheritance (e.g. Sykes, 1978; Audet \& Bürgmann, 2011).

Frictional plastic strain or viscous strain softening in lithospheric deformation models is often described by a linear decrease of the yield stress due to the accumulated viscous strain (more precisely the time integral of the second invariant of the strain rate tensor), which is motivated by field observations of possibly reduced strength and higher degree of localization of more mature faults (e.g. Lavier et al., 2000). The weakening and localization in different parts of the lithosphere is inferred to arise from different physical processes (e.g. Karato et al., 1986; Braun et al., 1999). However, frictional plastic strain or viscous strain softening does not consider the healing of weak zones and, as often implemented, cannot provide information on the time scale of tectonic inheritance or lithospheric break up.

On Earth and possibly other terrestrial planets the lithosphere thus contains weak zones which might be formed by different mechanisms (e.g. Karato, 1980; Bercovici, 1993; Zhong \& Gurnis, 1995b; Richards et 
al, 2001; Thielmann \& Kaus, 2012; Landuyt and Bercovici, 2009; Baes et al., 2001; Montési, 2013; Bercovici et al., 2015; Mazzotti \& Gueydan, 2018). Such preexisting weak zones can facilitate the breakup of the lithosphere to form new plate boundaries (e.g. Zhong \& Gurnis, 1996; Zhong et al., 1998; Landuyt et al., 2008) and initiate continental rifting. However, any form of preexisting weak zone or tectonic inheritance requires a certain form of strain memory or preservation of weak zones in the lithosphere. The formation and development of such weak zones (inherited intraplate or active plate boundary weak zones) in plate-like convection models and their effect on convection patterns and plate reorganization remains to be explored fully.

In summary, a range of mantle convection models have applied weakening mechanisms such as grain-size dependent rheologies, but uncertainties remain as to which mechanism is dominant, and which processes control the time scales of weakening and strain memory. Lithospheric models often employ ad hoc, straindependent rheologies which are motivated by field observations but are usually not linked back to general mantle rheology.

Here, we analyze the effects of a parameterized damage weakening rheology, strain-dependent weakening, on plate tectonic behavior and long-term convection dynamics in 2-D Cartesian thermal convection models. Our goal is to understand how such simplified (or parameterized) descriptions affect the transition between mobile and stagnant-lid convection states and the time-dependence of plate boundary formation, e.g. due to initiation of subduction zones, failed rift zones, or reactivation of inherited weak zones. We seek to use these models to gain insights into diagnostic plate tectonics memory behavior of an idealized, oceanic plate only system, and to find general parameters of weakening that can be used to constrain the correct microphysics in a next step. 


\section{Methods}

\subsection{Model Setup}

We analyze thermal convection models with a pseudo-plastic, temperature-dependent rheology in combination with strain-dependent weakening in a 2-D Cartesian model domain with an aspect ratio (width over height of domain) of eight. While some of the complexities of the plate-like style of convection (e.g. toroidal flow) can only be explored by 3-D computations, we find it useful to reduce complexity as a first step and here discuss simple, illustrative models.

The equations governing thermal convection in an incompressible, infinite-Prandtl number fluid (eqs. 1-3) are solved in the Boussinesq approximation using CitcomCU (Moresi \& Gurnis, 1996; Zhong, 2006) for a quasi 2-D Cartesian geometry ( 8 x $0.02 \times 1$ in $x,-y$,-and $z$-direction).

$u_{i, j}=0$

$-P_{, i}+\left(\eta u_{i, j}+\eta u_{j, i}\right)_{, j}+\operatorname{RaT} \delta_{i z}=0$

$T_{, t}+u_{i} T_{, i}=T_{, i i}+Q$

Here $u$ is the velocity, $P$ is the dynamic pressure, $\eta$ is the effective viscosity, $T$ the temperature, and $Q$ is the internal heat production rate. The term $X_{, y}$ stands for the derivative of $X$ in the direction of $y$, where $i$ and $j$ are spatial indices, $z$ is in the up direction, and $t$ represents the time. The system is heated only from within with a constant rate of non-dimensionalized $Q=10$, where we assume zero heat flux at the bottom and a constant temperature $(T=0)$ at the top. $R a$ is the bottom heated Rayleigh number defined as

$$
R a=\frac{\rho g \alpha \Delta T D^{3}}{\eta_{r e f} \kappa}
$$


where $\rho, g, \alpha, \Delta T, D, \eta_{r e f}$, and $\kappa$ are the density, gravitational acceleration, thermal expansion, temperature difference across the entire layer, depth of the layer, reference viscosity, and thermal diffusivity, respectively. We define $R a$ to be $10^{5}$ assuming values similar to Earth for the scaling parameters and a reference viscosity of $10^{23}$ Pas (see table 1 for additional scaling parameter values). The internal heating Rayleigh number which governs the effective convective vigor in our models is defined by

$$
R a_{Q}=\operatorname{Ra} Q
$$

and we explore variations of $R a_{Q}$. We use free slip velocity boundary conditions at the top and bottom and reflective boundaries at its sides. The resolution is 513 x 65 elements in $x$ - and $z$-direction and we use an initial number of 30 markers per element to track strain. At the top $(<0.1)$ and bottom $(>0.9)$ of the model domain, representing the area of highest interest, i.e. the lithosphere and the core mantle boundary (CMB), we use a grid refinement which provides twice the resolution as in the remaining mantle. Scaling parameters used in this study are defined and summarized in Table 1 . The resolution is sufficient to ensure a stable solution of eqs. (1-3). This is assured by resolution tests for refinement and shown by the surface heat flux for a mobile-lid convection which confirms energy conservation over time with an average surface heat flux corresponding to the internal non-dimensionalized heating rate (10). Higher resolution and higher number of markers do not significantly change the root mean square velocity, mobility, and plateness or strain amplitude.

\subsection{Rheology and strain-dependent weakening}

The temperature-dependent viscosity is described by an Arrhenius-type viscosity (e.g. Tackley, 2000a, b):

$$
\eta(T)=\eta_{0} \exp \left[\eta_{1}\left(\frac{1}{T+1}-\frac{1}{2}\right)\right]
$$


where $\eta_{0}$ is a non-dimensional pre-factor $\left(\eta_{0}=1\right), T$ is the non-dimensional temperature (scaled by the temperature difference $\Delta T$ ) and $\eta_{1}$ is the non-dimensional activation energy. We use a non-dimensional activation energy of 23.03 , which results in a temperature defined viscosity contrast of $\Delta \eta=10^{5}$ for a temperature range of zero to unity.

The strength of the material is defined by its yield stress (e.g. Tackley, 2000a, b; Enns et al. 2005):

$\sigma_{y, 0}=\min (a+b z, \lambda)$

where $a$ is the cohesion, $b$ is a depth gradient, which can describe a failure envelope for 'brittle' behavior in shallow depths, $z$ is the depth, and $\lambda$ is a constant yield stress for 'ductile' behavior. While the plate-like character of convection in 3-D is controlled by the definition of the yield stress (e.g. Tackley, 2000a), our 2-D thermal convection model setup showed that a depth-dependent yield stress does not lead to an improved plateness. As we are interested in how the strain-dependent weakening affects plate-like character and to avoid further complexities using additional parameters, we focus on models with a constant ductile yield stress.

Different strain-localization mechanisms have different potentials for weakening (e.g. Montési, 2013), and how their relevance works out for different parts of the Earth's mantle is debated. Rather than focusing on a specific mechanism, we describe the weakening to a general damage formulation depending on the accumulated viscous strain $\gamma$ to gain some first order understanding of the effect of convection, before linking things back to specific microphysical mechanisms. We note that the tracked strain $\gamma$ in our models is not the real strain (which cannot actually be removed, for example) nor a proper state variable, but rather an apparent, strain-dependent damage variable controlling the intensity of weakening. For the sake of convenience, we will refer to this apparent viscous strain variable $\gamma$ as "strain" in the following.

The temporal evolution of the strain is defined by 


$$
\frac{d \gamma}{d t}=\dot{\varepsilon}_{I I}-\gamma H(T)
$$

where the first term on the right-hand side is a source term given by the second invariant of the strain rate and the second term a temperature-dependent healing factor. The temperature-dependent healing rate is assumed to be an average of a possibly constant and purely temperature-dependent (e.g. due to diffusion processes) healing rate, which can be described by half the inverse of the diffusion creep viscosity (e.g., Tackley, 2000b):

$$
H(T)=B \exp \left[-\frac{\eta_{1}}{2}\left(\frac{1}{T+1}-\frac{1}{2}\right)\right]
$$

where $B$ is a constant describing the time scale of healing, i.e. assuming no active deformation is present, strain in the mantle $(T=1)$ is reduced by a constant rate of $B$. We assume $B$ to be on the order of 0.362 362.16 , which represents typical strain rates of the mantle of $10^{-13}-10^{-16} \mathrm{~s}^{-1}(B$ is scaled by the overturn time $t_{O T}$ and corresponds to 1/a number of overturns). The decrease in strain is governed by the healing rate $H$, which is defined by the temperature and the healing time scale. For example, assuming deformation is not active, i.e. strain rate is equal to zero, eq. (8) is defined as an exponential decay. The time to reduce strain by a factor of $1 / \mathrm{e}$ is inverse proportional to the healing rate $H$ (Fig. 1). Thus, within the mantle $(\mathrm{T}=1)$ strain is removed fast $(<1 \mathrm{OT})$ even for low healing time scales of $B=1$. In the lithosphere, however, time to reduce strain increases significantly for low healing time scales and can be preserved up to 100 OT, whereas it remains $\sim 1$ OT only for high healing time scales of $B=362.16$. This healing mechanism mimics a reduction of the effective strain either by mixing and stirring mantle processes with typical strain rates of the mantle or due to temperature dependent diffusion processes (e.g. grain growth). The time-scales for strain reduction does, on average, match the time scale of grain growth measurements. While temperaturedependent healing allows us to avoid infinite strain accumulation, it also permits long term strain memory in the cold lithosphere and healing within the mantle. 
The weakening, i.e. the effective yield stress, is defined by a linear reduction of the yield stress due to the accumulated strain $\gamma_{c r}$ (e.g. Lavier et al., 2000; Huismans \& Beaumont, 2003; Mazzotti \& Gueydan, 2017):

$$
\tilde{\sigma}_{y}(t)=\sigma_{y, 0}\left[1-D_{\max } \frac{\gamma(t)}{\gamma_{c r}}\right]
$$

where $\gamma_{c r}$ is a critical strain and $D_{\max }$ a maximum 'damage' of $90 \%$. The maximum defined damage $D_{\max }$ results in a maximum reduction of the yield stress by a factor of 10, similar to previous results found in lithospheric work (cf. Gueydan et al., 2014). While a linear decrease of the yield stress is often used in models to localize strain in brittle material (e.g. Lavier et al., 2000; Huismans et al., 2005), an exponential decrease seems to be appropriate for a non-linear, power-law viscous material (e.g. Gueydan et al., 2014). However, since we assume a linear rheology, the strength drop between the deformed and undeformed material, rather than the rate of reduction, is the controlling parameter regarding strain localization (Mazzotti \& Gueydan, 2018) and time scales of tectonic inheritance.

According to eq. (10), we assume that the accumulated strain leads to weakening of the material by reducing the yield stress whereas plastic failure only occurs if the local stress exceeds the reduced yield stress. The accumulated damage can then be defined simply by:

$$
D=1-\frac{\tilde{\sigma}_{y}}{\sigma_{y, 0}}
$$

Following this description, the weakening is controlled by: a) the healing time scale $B$ expressing how long strain can be preserved and $b$ ) the critical strain $\gamma_{c r}$. Assuming a constant strain rate over a given time, strain reaches its maximum faster for high healing time scales and due to the lower maximum strain weakening is less effective in comparison to low healing time scales (Figs 2a and c). A small critical strain leads to a 
fast, and thus more effective, rate of weakening in comparison to high critical strains (Figs $2 \mathrm{~b}$ and c). This weakening formulation is assumed to appropriately mimic more complex rheological weakening mechanisms (like grain-size dependent rheology), at least for the first order behavior.

The variation in effective viscosity due to the strain-dependent weakening formulation from eq. (10) using a range of $\gamma_{c r}(0.1-18)$ and $B(0.362-362.16)$ correlates with the variation in the effective viscosity due to a grain-size dependent, composite, effective rheology (e.g., Solomatov, 2001; Braun et al. 1999; Rozel et al. 2011; Dannberg et al., 2017) for a range of temperatures $\left(300-900{ }^{\circ} \mathrm{C}\right)$ and strain rates $\left(10^{-13}-10^{-16} \mathrm{~s}^{-1}\right)$. While the absolute variation in the effective viscosity due to a grain-size dependent rheology can be matched by the strain-dependent weakening, the rate of the variation and a more accurate fit between both depends on more parameters (e.g. kind of grain-size evolution model, transition between dislocation and diffusion creep). A detailed comparison between different weakening deformation mechanisms and the strain-dependent weakening rheology is beyond the scope of this paper but will be addressed in a second paper.

The yield and effective viscosity are defined as (e.g. Tackley, 2000a, b):

$$
\begin{aligned}
& \tilde{\eta}_{y}=\frac{\tilde{\sigma}_{y}}{2 \dot{\varepsilon}_{I I}} \\
& \eta_{e f f}=\min \left(\eta_{T}, \tilde{\eta}_{y}\right) .
\end{aligned}
$$

Due to a temperature higher than unity and the effect of yielding, effective viscosity can be much smaller than unity. To avoid numerical difficulties, we confined the minimum viscosity to be $10^{-2}$, which result in a maximum total viscosity difference of $10^{7}$ within the entire model domain. 


\subsection{Diagnostics}

Plate-like behavior and long-term dynamics of the convecting system can be quantified by the mobility $M$ and the plateness $P$ (e.g. Tackley, 2000a). Mobility is defined as the ratio between the root mean square velocity at the surface $v_{r m s, \text { surf }}$ and the entire mantle $v_{r m s, w h o l e}$ :

$M=\frac{\left(V_{R M S}\right)_{\text {surf }}}{\left(V_{R M S}\right)_{\text {whole }}}$.

Here, plateness is defined by 1 minus the area covering $80 \%$ of the maximum strain rate at the surface (e.g., Tackley, 2000a; Foley \& Becker, 2009):

$P=1-f_{80}$

In case of an isoviscous or stagnant-lid convection, the strain rate at the surface and thus plateness is not equal to zero. For an isoviscous or stagnant-lid convection with $R a_{Q}=10^{6}$, for example, plateness is $\approx 0.51$ and $\approx 0.37$ and mobility is $\approx 1.15$ and 0 , respectively. Assuming motion at the surface is close to the theoretical approximation of plate tectonics, in which deformation is perfectly localized along the plate boundaries, plateness approaches unity. Based on the plateness of a 2-D stagnant-lid convection, here, we define the minimum plateness, or an at least plate-like convection, to be $\approx 0.37$.

To better understand and quantify the effect and importance of the strain-dependent weakening parameters $B$ and $\gamma_{c r}$, and the history of deformation on plate-like convection and reorganization of plate boundaries, we first conducted a detailed sweep through the $B(0.36-362.04)$ and $\gamma_{c r}(0.94-18)$ parameter space for a constant Rayleigh number and yield stress, i.e. the reference model $\left(R a_{Q}=10^{6}\right.$ and $\left.\sigma_{y, 0}=4 \cdot 10^{3}\right)$. We analyzed long-term convection dynamics of these models during a given model period of 40 overturn times (OT), which corresponds to a time of $\sim 4.5$ billion years for an Earth-like overturn time assuming an average 
plate velocity of $\sim 5 \mathrm{~cm} / \mathrm{a}$. One overturn time for each model is defined by the ratio of two times the thickness of the mantle and a characteristic velocity, i.e. the time average of the root mean square velocity (see Table 1).

Besides the mobility and plateness, we calculated the surface-averaged strain, $\gamma_{\text {surf }}$, and yield stress, $\sigma_{y, s u r f}$, to measure their temporal evolution at the surface. The time average of those surface-averaged metrics provides an estimate on the average surface strain (ASG) and damage (ASD; see eq.(11)). In addition, we analyzed the time average of the mobility and plateness, as well as the relative mobility, i.e. the time the mobility is above 0.1 , which we define as the transition between a mobile and stagnant-lid convection.

Second, we analyzed how strain-dependent weakening, in general, affects the transition between mobile and stagnant-lid convection. We run a series of models without strain-dependent weakening in the $R a_{Q}-\sigma_{y, 0}$ parameter space to map this boundary. We choose certain models (see rectangles in Fig. 3a) close to the stagnant-mobile lid transition of each $R a_{Q}$ as initial condition, increased the yield stress $\sigma_{y, 0}$ step wise, i.e. $R a_{Q}=5 \cdot 10^{5}, \sigma_{y, 0}=2 \cdot 10^{3}, 3 \cdot 10^{3}, 4 \cdot 10^{3} ; R a_{Q}=10^{6}, \sigma_{y, 0}=4 \cdot 10^{3}, 5 \cdot 10^{3} ; R a_{Q}=5 \cdot 10^{6}, \sigma_{\mathrm{y}, 0}=2 \cdot 10^{4}$, and included strain-dependent weakening. For each of those models, we run 6 additional models with specific straindependent weakening parameters $\left(B=1.81,181.02 ; \gamma_{c r}=10,3.6,2\right)$, representative of the characteristic features based on the detailed sweep conducted at first. Each of those models run for a time of at least 100 OT. A model is considered to be mobile if the mean of the mobility over the full model time is less than 0.1 (large diamonds in Fig. 11).

To quantify the effects of strain-dependent weakening on the mobile-stagnant lid transition, we calculated the time-averaged surface-average damage (ASD) and the relative difference in mean yield stresses in the lithosphere over the longest mobile-lid period or at least a mobile-lid time period of 40 OT. The relative difference in the mean yield stresses is defined by a) the relative difference between the mean yield stress 
at the surface and the base of the lithosphere (i.e. a depth of 0.05) $\Delta \sigma_{\text {mean }}$, b) the relative difference between the mean yield stress at the surface and its minimum $\Delta \sigma_{\text {surf-min }}$, and c) the relative difference between the mean yield stress at the base of the lithosphere and its minimum $\Delta \sigma_{L B-m i n}$. The first describes the average weakening of the lithosphere with depth (a) and the latter two describe the intensity of the weakest weak zone at the surface (b) and how strong it penetrates through the lithosphere (c).

\section{Results}

\subsection{Strain accumulation and surface strain - no weakening}

To understand the general systematics and how the strain $\gamma$, the surface-averaged strain $\gamma_{\text {surf }}$, and the timeaveraged surface-average strain ASG evolve in our 2-D thermal convection models, we first focus on models without strain-dependent weakening before including the weakening feedback. Each model starts with a thermal perturbation as initial condition and runs until a statistical steady state is reached. As discussed in section 2.2, the strain amplitude is controlled by two mechanisms, the accumulation rate, governed by the local strain rate, and the overall healing rate $H$, governed by healing time scale and temperature. The default healing time scale for strain accumulation in models without strain-dependent weakening is set to 3.6216 corresponding to an average strain rate of the Earth's mantle (see section 2 for scaling). We would like to note, that assuming a different healing time scale would result in different strain amplitudes. However, as the healing time does not depend on the actual amount of strain (Fig. 1), the timescales of strain do not vary with varying $B$. To highlight the time-dependent behavior of the abovementioned strain parameters, we choose a reference model for an intermediate $R a_{Q}$ and $\sigma_{y, 0}$ resulting in a weakly episodic lid (i.e. $R a_{Q}=10^{6}$ and $\sigma_{y, 0}=4 \cdot 10^{3}$ ) and discuss the main results below in detail. The described dynamics and characteristics controlling the temporal and spatial evolution of strain in the reference model are the same as in models with a different $R a_{Q}$ and $\sigma_{y, 0}$, 
The mobile-lid stage of the reference model is dominated by double-sided downwellings ("subduction zones") initiated at the surface due to yielding (Fig. 3a, top), as has been explored in a range of studies before. As the cold slabs sink in to the mantle, divergent zones (upwellings) are formed at the surface thinning the lithosphere and forming "spreading centers". Yielding at the surface occurs along two oblique bands within the upper thermal boundary layer ("lithosphere") and is usually initiated at locations where the lithosphere is thickest. One plastic weakening band defines the "plate" boundary between the overriding and subducting plate and one weakening band lies in the bending region of the "slab". The weakening in the bending region is extensive, implying that our model is largely plastically deforming, and stronger slab cases might behave differently. However, we note that slabs appear quite weak in nature (e.g. Billen \& Hirth, 2007; Becker \& Faccenna, 2009), and Holt et al. (2015) suggested that significant plastic yielding in the slab bending region might be reflected in slab curvature systematics as on Earth.

The strain field, which governs the local rheological weakening in subsequent presented models, is strongly governed by those dynamics as well (Fig. 3a, bottom). Subduction zones are characterized by high strain accumulation at the surface, due to the large-scale pure shear type deformation. As the slab sinks into the mantle, strain is advected but decreases slightly due to increasing temperature (leading to healing/reduction of strain) and less intense deformation inside the slab. Within the lower mantle strain increases again due to vertical compression. However, as the slab is heated over time and active deformation ceases, strain is removed entirely within the lower mantle. Due to the spreading centers and the cold lithosphere, strain within the upper thermal boundary layer $(z<0.1)$ is not equal to zero, resulting in a finite strain value directly at the surface. In the lithosphere, strain decreases with depth due to an increased healing rate with increasing temperature and weaker shear. Directly below the lithosphere, strain slightly increases again due to a stronger active shear before being almost completely reduced in the mantle. 
The surface-averaged strain, $\gamma_{\text {surf }}$, is strongly governed by those dynamics (solid line in Fig. 3b). The surface-averaged strain decreases slightly when convection slows down and increases slightly when convection accelerates again. Due to the spreading centers and convergent plate boundaries governed by the convection dynamics, $\gamma_{\text {surf }}$ oscillates around a time-independent constant strain value, i.e. the timeaveraged surface-average strain $/ \gamma$ (ASG - dashed line in Fig. 3b). The ASG is mainly governed by the average strain accumulated in the lithosphere due to the average number of spreading centers and convergent plate boundaries. So, when new subduction zones are formed or die, the surface-averaged strain oscillates around the ASG. The surface-averaged strain varies strongly only in case a stagnant-lid is formed or broken up. Given a long enough stagnant-lid phase, depending on the healing time scale $B$ (see Fig. 2), strain could even be reduced entirely within the lithosphere. Thus, the time scale of $\gamma_{\text {surf }}$ is controlled by the frequency of the formation and destruction of plate boundaries, the stability of the plates, and the healing time scale $B$.

As the surface-averaged strain is governed by convection dynamics and formation of plate boundaries, $\gamma_{\text {surf }}$ shows a general correlation with the mobility and plateness (Fig. 3c). As convection slows down mobility decreases, and a lid is formed, reducing plateness accordingly. The stagnant lid leads to an increase in internal temperature and velocity, which results in local stress concentrations in the lithosphere, high enough to overcome the yield stress. With the breakup of the lithosphere, mobility increases again as well as plateness. Mobility and plateness vary over a broad range where the medians are $\approx 1.24$ and $\approx 0.71$, respectively.

In case the model is in a pure mobile-lid state and assuming a constant healing time scale, the ASG is controlled by the parameters controlling convection, i.e. $R a_{Q}$ and $\sigma_{y, 0}$ (Fig. 4 b). Thereby, the ASG decreases with increasing yield stress, as the model moves towards the stagnant-lid boundary. The formation of less numerous spreading centers and convergent plate boundaries due to the higher $\sigma_{y, 0}$ and thus stronger plates 
leads to a smaller ASG. Increasing $R a_{Q}$, however, results in an increase of the ASG, as the root mean square velocity increases as well, which results in higher strain rates along the ridges and convergent plate boundaries and thus a higher strain amplitude.

\subsection{Strain-dependent weakening and deformation history}

In the following we discuss the effect of the strain-dependent weakening rheology on plate reorganization and convection patterns. First, we focus on the effect of the strain-dependent weakening parameters $B$ and $\gamma_{c r}$ applying strain-dependent weakening to a reference model $\left(R a_{Q}=10^{6}, \sigma_{y, 0}=4 \cdot 10^{3}\right)$, before expanding the analysis to the entire $R a_{Q}-\sigma_{y, 0}$ parameter space. A mobile-lid stage of the reference model is used as initial condition for models including strain-dependent weakening. For each model we systematically varied the critical strain $\gamma_{c r}$ and healing time scale $B$ and ran it up to 100 OT. Models including strain-dependent weakening do reach statistical steady state after 5-10 OT. To analyze long-term effects of strain-dependent weakening on the convection patterns and plate configurations, we used the $10^{\text {th }} \mathrm{OT}$ as a start and the $50^{\text {th }}$ OT as an end, providing a total analysis time of $\sim 40$ OT.

In our strain-dependent weakening models we can track the self-consistent formation and reactivation of weak zones in the lithosphere, both inherited intraplate as well as active weak plate boundaries (Fig. 5). Those weak zones are characterized by high strains and low yield stresses and form as remnants of largescale pure shear type deformation at subduction zones. Depending on their location with respect to the convecting interior and intensity, i.e. the damage $D$ (see eq.(11)) and depth extension, they can be reactivated in different manners. The most common forms of reactivation are intraplate subduction initiation, ridge adjacent subduction initiation, and spreading center initiation. Besides localized dominant weak zones, the yield stress in the upper part of the lithosphere is reduced by the ASG. In the following we present some examples of such reactivation processes from our $B-\gamma_{c r}$ parameter space analysis in more 
detail. We consider these examples as useful for a mechanical understanding of processes that might be evolved in the Earth's Wilson cycle, for example.

In the subduction zone, high strain is accumulated due to the large-scale pure shear type deformation at the convergent plate boundary (the "trench", $t=3.5$ OT in Fig. 5b). When the slab breaks off ( $t=4.8$ OT), the strain in the former trench region is preserved and forms a weak zone with a reduced yield stress in a new continuous plate. Depending on the weakening parameters $B$ and $\gamma_{c r}$, damage in the lithosphere can vary due to the strain amplitude. Within the actively deforming trench, however, damage can reach a maximum of $90 \%$, especially for models with a small critical strain. As the plate moves laterally due to an active adjacent subduction zone, the inherited weak zone is advected laterally with the plate $(t=5.8 \mathrm{OT})$. If the effective yield stress in the weak zone is smaller than the surrounding average lithospheric yield stress (which is governed by the ASG shown in Fig. 5c bottom), the weak zone can be reactivated $(t=6.4$ OT) and forms a new subduction zone, here after a preservation time of $\approx 1.6$ OT. If the weak zone is not reactivated, it will be subducted along the dominant active subduction zone driving the plate or by a newly initiated, adjacent subduction zone.

The surface-averaged strain $\gamma_{\text {surf }}$ and surface-averaged effective surface yield stress $\tilde{\sigma}_{y, \text { surf }}$ are in direct anticorrelation to each other, while the yield stress increases, strain decreases. A peak in $\gamma_{\text {surf }}$ indicates a new formation of a subduction zone while a trough indicates a reduction of the total numbers of active subduction zones and more effective healing. For example, between $t=3.5$ and 4.8 OT, mobility increases due to the active subduction zones accelerating the plates at the surface and the surface-averaged strain decreases due to more effective healing in the moving, non-deforming plates. From the moment of the slab break off $(t=4.8 \mathrm{OT})$ to the initiation of the intraplate subduction $(t=6.4 \mathrm{OT})$, the surface-averaged strain decreases further. Due to the newly formed subduction zones and ridges, the surface-averaged strain increases again. 
Mobility and plateness anti-correlate as well, while an increase in plateness, i.e. localization of active deformation within a narrower area, is accompanied by a decrease in mobility (Fig. 5c, top). Interestingly, this shows an opposite correlation to the observation for models without strain-dependent weakening. The anti-correlation between mobility and plateness is governed by an enhanced localization along the plate boundaries due to weakening. The time-dependent behavior is related to the formation of subduction zones, as well as spreading centers. If scattered subduction zones dominate the overall convection, or in the event of a newly formed subduction zone, plateness increases as deformation is focused along the trenches. The sinking slabs increase the overall velocity in the mantle with respect to the velocity at the surface and mobility decreases. As the plates move laterally, dragged by the sinking slabs, mobility increases while plateness decreases due to the formation of broad divergence zones at the surface.

The presence of lithospheric weak zones, however, does not always dictate a failure along those weak zones (Fig. 6). While a weak zone is formed at the surface (Fig. 6a, $t=1.4$ OT) and advected laterally (Fig. 6b, $t$ $=2.06 \mathrm{OT})$, failure is not initiated at the location of the weak zone itself $(t=2.46 \mathrm{OT})$. Although the healing time scale and critical strain are the same as in the previously presented example and time between the slab break off and subduction initiation is shorter $(\approx 1$ OT), the inherited weak zone is not reactivated. Instead, a new subduction zone is formed adjacent to the inherited weak zone. The intensity of the weak zone is similar to the previously described example $(\gamma \geq 5)$, which is about twice as large as the ASG (Fig. 6c). But, the location to the adjacent spreading center $(x=6)$ is too far with respect to the size of the convecting cell. Thus, the lithosphere yields adjacent to the inherited weak zone which is not reactivated. Plateness, mobility, and surface-averaged metrics behave in an equivalent manner as previously described. However, the surface-averaged effective yield stress slightly decreases from the moment the slab breaks off ( $t=2.06$ OT) to the new subduction initiation $(t=2.46$ OT). The overall lithospheric weakening could additionally facilitate failure of the lithosphere away from the inherited weak zone. 
Besides convergent plate boundary formation, tectonic inheritance can also be reactivated as a spreading center (Fig. 7a and b). Again, the weak zone is formed as a remnant of a subduction zone $(t=0.58$ OT) and advected laterally with the motion of the plate $(t=2.21 \mathrm{OT})$ over a period of $\approx 3$ OT. Due to the low healing time scale (0.3622) it is fully preserved. At a later point, a new subduction zone is formed adjacent to the inherited weak zone. The drag of the subduction zone on the plate in the positive $x$-direction forms a new spreading center at the location of the weak zone. Mobility, plateness, and surface-averaged metrics behave as previously described (Fig. 7c). Due to a higher critical strain, the effective surface yield stress is higher in comparison to the previous examples with the same healing time scale (cf. Figs 4 and 5) although the strain has the same order of magnitude. This results in a slightly less effective strain-dependent weakening.

Consequently, the reactivation of a weak zone is governed by two main aspects: a) the intensity of the weak zone, i.e. the effective yield stress amplitude and the depth extension, as well as b) the location of the weak zone with respect to the convecting interior or the size of the convecting cell. This has mainly been observed visually by analyzing examples of reactivated and non-reactivated weak zones. The intensity of the weak zone is controlled by the healing time scale $B$, which allows its preservation in the lithosphere, and the critical strain, which defines the amount of strain required to obtain maximum damage. The healing time scale also has a second order effect on the location, as $B$ determines the time a weak zone can be advected laterally with the plate before being reduced significantly.

The formation of new plate boundaries (convergent or divergent) adjacent to a prior initiated plate boundary (divergent or convergent), also occurs in models without strain-dependent weakening. However, without strain-dependent weakening, the distance of the newly formed plate boundary adjacent to the prior initiated plate boundary varies strongly. Instead, tectonic inheritance adjacent to the prior initiated plate boundary pre-determines the location of the newly formed plate boundary. 


\subsection{Long-term dynamics}

Allowing for strain-dependent weakening in mantle convection leads to different plate boundary dynamics relative to pure pseudo-plastic convection and affects the long-term behavior of the planform convection. Depending on the healing time scale and the critical strain, weakening becomes less or more effective (Fig. 8). To quantify the long-term effect of strain-dependent weakening parameters on plate reorganization and stability, we analyzed the time-averaged surface-average metrics (ASG and ASD), the mobility and plateness, as well as the root mean square velocity and relative mobility within the $B-\gamma_{c r}$ parameters space.

The over-all temperature-dependent healing rate $H$, and thus the healing time scale $B$, partly controls the amplitude of the ASG (Fig. 8a). The strain, in combination with the critical strain, defines the timeaveraged surface-average damage (ASD, Fig. 8b). Besides the inherited weak zones, the ASD contributes to the convecting behavior, as it approximates the effective surface yield stress and thus governs yielding within the shallow part of the lithosphere. With decreasing critical strain, ASD increases, while the increase is more effective for low in comparison to high healing time scales. Due to a faster healing, the moving plates carry a smaller amount of strain. Therefore, the ASD is almost negligible $(<1 \%)$ for high healing time scales $(B>100)$. Within this range, the convective behavior is similar to a rheology without straindependent weakening.

For a small critical strain, however, less accumulated strain is required to obtain weakening, which results in more localized weakening events similar to a strain-rate/pseudostick-slip weakening rheology. Due to more effective weakening and higher damage at smaller strains along active plate boundaries in models with a small critical strain, a once active subduction zone tends to dominate surface motions. That is, even in the case of a slab break off and the formation of a tectonic inheritance, reactivation is never observed for small critical strains as the surface motion is focused on the active subduction zone. Strain memory also becomes less effective for higher healing time scales, which results in the absence of any reactivation of 
weak zones. Reactivation of inherited weak zones has been observed for models with a critical strain $\gamma_{c r} \geq$ 6 and low to intermediate healing time scales $(B \leq 3.6216)$. The present weak zones result in stress localizations which facilitate failure in the lithosphere. As no subduction initiation is observed at the location of a high strain zones in models without strain-dependent weakening, it is most likely, that yielding is controlled by the inherited weak zone.

Mobility and plateness are only slightly affected in cases of low $(<20 \%)$ ASD (Figs 7c and d). While mobility is within the range of the reference model $\left(M_{r e f} \approx 1.19\right)$, plateness is slightly increased due to straindependent weakening $\left(P_{\text {ref }} \approx 0.72\right)$. With decreasing healing time scale and critical strain, i.e. increasing ASD, plateness increases further due to a more localized deformation in the lithosphere. For a high average deformation (ASD > $60 \%$ ), due to small critical strains and healing time scale, weakening becomes too effective and deformation is strongly localized at the surface. The intense weakening of the lithosphere results in a breakdown of plate-like tectonics into more drip-like tectonics. Mobility is reduced for high ASD as the slabs or drips are not strong enough to drag the plates laterally along the surface and motion is concentrated only within a small area. The effect of the strain-dependent weakening and the resulting stronger localization along the plate boundaries is also observable in the time-averaged root mean square velocity (Fig. 9a). The strong overall weakening (high ASD), as well as the strong localization and the lithospheric drips, result in a strong increase in the root mean square velocity. This shows the range where plate-like convection starts to break down. For low to intermediate ASD, the root mean square velocity is not significantly affected by strain-dependent weakening.

More important though, is the effect of strain-dependent weakening on the time convection is in a mobilelid phase, i.e. the relative mobility (Fig. 9b), and the stability and longevity of plates in the mobile-lid stage (Fig. 10). In cases of a low ASD, relative mobility varies strongly with short periods of stagnant-lid phases, similar to the reference model. However, most of the models with low ASD tend to have a high relative 
mobility of up to $100 \%$, i.e. convection never enters a stagnant-lid. The relative mobility tends to be smaller in cases of high healing time scales $(B>36.216)$, whereas convection stays in mobile-lid or relative short stagnant-lid phases for lower healing time scales mainly due to reactivation of weak zones and the weakening along plate boundaries. With decreasing critical strain, weakening becomes more effective which results in fully mobile-lid convection for ASD $>20 \%$. Therefore, due to the tectonic inheritance and their reactivation, as well as the overall weakening of the lithosphere, strain-dependent weakening allows convection to be mobile over a longer period, although the initial yield stress of the lithosphere tends to result in a weakly episodic-lid convection.

As shown by the increase in relative mobility, including strain-dependent weakening increases the longevity of mobile-lid stage and stability of a plate-like convection, due to the reactivation of weak zones and strain localization along active plate boundaries. Considering only the mobile phase of those models, an increase in stability of plate-like convection is also visible in a shift to higher dominant periods in the time series of the total heat flow at the surface (Fig. 10). The time series of the total heat flow represents the formation and destruction of new plate boundaries, i.e. total heat flow increases and decreases depending on the average active number of plate-boundaries and plate area. A shift of the dominant period of the total heat flow at the surface to higher periods, shows less variability in the formation and destruction of plate boundaries and more stable plate-like convection due to strain-dependent weakening. With increasing critical strain, i.e. more effective weakening, and for high healing time scales, the dominant wavelength increases. This has also be observed in the time variability of the average number of active convergent plate boundaries for models with and without strain-dependent weakening. The amplitude of the time the system has a certain number of active subductions does not show a clear correlation including strain-dependent weakening. However, its variation, i.e. the stability of a certain amount of active convergent plate boundaries, decreases in models with strain-dependent weakening. 
In general (Figs $8 \mathrm{~b}$ and 9b), strain memory becomes less important for high healing rates and high critical strains (or very low ASD $<5 \%$ ). With decreasing $B$ and $\gamma_{c r}$ (or low ALD $<20 \%$ ), strain memory becomes more important and reactivation of tectonic inheritance is observed. For intermediate ASD (20 - $60 \%)$, weakening is most effective, resulting in a high plateness, low mobility, and fully mobile-lid phase. For high ASD (> $60 \%)$, weakening becomes too effective resulting in a significant drop of the average effective surface yield stress, low mobility, and strong localization of deformation at the surface $(P>0.85)$. In this case, convection becomes unstable due to the eventually strong weakening of the lithosphere and entire mantle and motion is mainly controlled by drip-like tectonics. Including strain-dependent weakening results in a higher longevity of a mobile-lid convection and increases its stability.

\subsection{Mobile-stagnant lid transition}

As shown by the reference model, including strain-dependent weakening results in a shift of the mobile-lid stage into regions of higher yield stresses. This shift can also be observed for cases with different $R a_{Q}$ and $\sigma_{y, 0}$ (Fig. 12). To compare models including weakening with models without weakening, we calculated the effective surface yield stress $\tilde{\sigma}_{y, \text { surf }}$ for each model by averaging the effective yield stress over the surface. Thus, the data points for models including weakening and an initial yield stress $\sigma_{y, 0}$, which would result in a stagnant-lid without weakening, fall into a lower effective yield stress range. The shift to lower effective yield stresses, however, is only significant for an average surface damage (ASD) of $>50 \%$ (compare $R a_{Q}$ and $\tilde{\sigma}_{y, \text { surf }}$ plots in Fig. 12a). Depending on the weakening parameters $B$ and $\gamma_{c r}$, strain-dependent weakening shifts the mobile-stagnant lid transition to slightly higher effective surface yield stresses, especially for low to intermediate $R a_{Q}$ cases (Fig. 11).

As the model starts in a mobile-lid stage, weakening along active plate boundaries and reactivation of weak zones in the lithosphere ensures an increase in the longevity of the mobile-lid stage. Weakening along the plate boundaries seems to be most important, as models with a small critical strain tend to be mobile for the 
entire model time (see $B \cdot \gamma_{c r}$ plot in Fig. 12a). With increasing $R a_{Q}$ and $\tilde{\sigma}_{y, \text { surf }}$, convection is only mobile in case the time-averaged surface-average damage is high enough ( $>20 \%$ ). Such a high damage, however, can only be reached if the critical strain $(<3.6)$ and healing time scale $(<1.81)$ are small enough. Each mobile-lid convection with a smaller damage has either a small $R a_{Q}$ and initial yield stress, which would result in a mobile-lid anyway (triangle markers in Fig.12), or a small critical strain (compare figures in Fig. 12a). As seen in the relative stress difference in the lithosphere (see $\Delta \sigma_{L B-\min }$ plot in Fig.12b), a small critical strain results in a relative difference between the mean effective yield stress at the base of the lithosphere and its minimum of $\sim 20-50 \%$, which ensures a mobile-lid convection for models with a small ASD. For the highest here applied $R a_{Q}$ which results in a mobile-lid convection, the time-averaged surface-average damage is at least greater than $40 \%$, which can only be reached for small critical strain and healing time scales.

As seen by the ASD, convection stays in a mobile-lid stage if the average surface damage is high enough. But more importantly, the relative difference in the yield stress in the lithosphere (in per cent) shows that the weak zone needs to penetrate through the entire lithosphere and needs to be relatively weak in case convection remains mobile-lid. With increasing effective surface yield stress the intensity of the weak zones needs to be higher as well $(>60 \%)$. The relative difference between the mean effective yield stress at the surface and the base of the lithosphere (see $\Delta \sigma_{\text {mean }}$ plot in Fig. 12b), as well as the relative difference between the mean effective yield stress at the surface and its minimum (see $\Delta \sigma_{\text {surf-min }}$ plot in Fig. 12 b) do not show a clear transition between a mobile and stagnant-lid convection for cases including strain-dependent weakening.

Therefore, the average relative weakening within the lithosphere $\left(\Delta \sigma_{\text {mean }}\right)$, as well as the relative weakening of a weak zone directly at the surface $\left(\Delta \sigma_{\text {surf-min }}\right)$, do not seem to be the dominant mechanism to provide a mobile-lid convection for initial $R a_{Q}-\sigma_{y, 0}$ parameters falling into the stagnant-lid regime. The relative 
difference between the mean effective yield stress at the base of the lithosphere and its minimum $\left(\Delta \sigma_{L B-\min }\right)$, which indicates a) if a weak zone fully penetrates through the lithosphere and b) the intensity of such a weak zone at the base of the lithosphere, seems to be more important. Each mobile-lid convection with initial $R a_{Q^{-}} \sigma_{y, 0}$ parameters falling into the stagnant-lid regime and including strain-dependent weakening has a weak zone fully penetrating through the lithosphere and a relative intensity of the weak zone with respect to the base of the lithosphere of at least $20 \%$ (see diamond markers in $\Delta \sigma_{L B-\min }$ plot in Fig. 12b). Moreover, the intensity of the weak zones increases with increasing effective surface yield stress, i.e. with an increasing $R a_{Q}$.

In general, a mobile-lid convection for model parameters with an initial $R a_{Q^{-}} \sigma_{y, 0}$ falling into the stagnantlid regime and including strain-dependent weakening does only occur for small critical strains $(<3.6)$ and healing time scale $(<1.81)$. Such small weakening parameters allow a weak zone to fully penetrate through the lithosphere and a relative intensity of the weak zone at the base of the lithosphere of at $20 \%$. The intensity of the weak zone and its depth penetration seem to be more important than the average weakening of the lithosphere, considering a mobile-lid convection and a shift of the stagnant-mobile lid transition to higher effective surface yield stresses.

Our models indicate, however, that in case convection reaches a stagnant-lid for high initial yield stress $\sigma_{y, 0}$, convection tends to stay stagnant for the remaining model period and does not become episodic, even for very small healing time scales. This has also been observed for test models starting in a stagnant-lid convection including strain-dependent weakening. Those models showed that convection does not become an episodic-lid, as the weakening results in a less viscous asthenosphere and a more effective decoupling between mantle and lithosphere, which would impede failure of a stagnant-lid. Only in cases of a small critical strain, convection stays mobile due to enhanced weakening along active plate-boundaries. However, in the $R a-\sigma_{y, 0}$ parameter space including weakening, we did not apply the minimum healing time scale from 
the first parameters sweep $(B=0.36)$, which could lead to a reactivation of weak zones in an episodic-lid convection.

\section{Discussion}

On Earth, and possibly other Earth-like planets, the lithosphere consists of shear-zones providing zones of a relative rheological weakness governed by a general damage. Those weak zones are formed by different tectonic mechanisms, like sutures of former subduction zones (e.g. Dewey, 1977; Buiter \& Torsvik, 2014), old orogenic belts (e.g. Butler et al. 2006; Mouthereau et al., 2013), transform or strike-slip faults (e.g. Baes et al., 2011) or failed rift systems (e.g. Sykes, 1978). If plate tectonics is active or has been active in the past, such weak zones could be critical for remobilization of the surface, assuming the weak zones are preserved over an extended period. While the main driving mechanism of subduction initiation remains debated, models show that subduction can be reinitiated along such rheological weak zones (e.g. Baes et al., 2011). However, those weak zones are usually imposed and do not form self-consistently and failure along such weak zones might be partially predetermined by applied boundary conditions or the model geometry. As our models show, the reactivation of tectonic inheritance in the lithosphere significantly depends on the properties of the weak zone, i.e. its damage intensity relative to the average lithospheric damage, its depth, and its location relative to the convecting interior.

Besides reactivation of subduction zones along inherited lithospheric weak zones, the ability to preserve weak zones over geologically long periods has been shown to be crucial regarding the onset of present-day plate tectonics or the reorganization of plates. As proposed by Bercovici \& Ricard (2013, 2014), two-phase grain size damage in combination with pinning is critical to provide a long-term preservation of tectonic inheritance in the lithosphere. Based on this theoretical approach, these authors proposed that lithospheric weak zones during the early Earth might be formed as remnants of time dependent, intermittent, and wide spread low-pressure zones (imposed as an approximation of subduction zones). When those widespread 
and intermittent sinks are shut off, they form lithospheric weak zones due to an increased damage which can be preserved over a sufficient geological period ( $1 \mathrm{Gyr})$. Such weak zones can be reactivated if new subduction zones are formed, which could lead to the initiation of present-day plate tectonics and plate-like surface motions with strike-slip transform faults (Bercovici \& Ricard, 2014).

As our weak zones are formed as remnants of subduction zones and strain can be preserved over sufficiently long geological times ( 10 OT, which corresponds to 1.1 Gyrs for Earth-like conditions), 3-D spherical convection models in combination with a strain-dependent damage weakening could lead to similar surface dynamics as observed by Bercovici \& Ricard (2014). Our preliminary global convection models in combination with strain-dependent damage weakening show a high longevity and stability of subduction zones due to strain localization and weakening.

Considering tectonic inheritance to be a major aspect in plate tectonics, the cyclicity of supercontinent cycles is strongly controlled by how well and how long the lithosphere preserves such weak zones. Here, we do not include continents, of course, but our assumption is that the general mechanisms explored in our approximate, oceanic-plate only convective system will be relevant for the combined system and to be explored next.

Our models show, that strain in the lithosphere can be preserved for at least $10 \mathrm{OT}$, if the healing time scale is low enough $(B \leq 3.6216)$. Such a long preservation might be not relevant for oceanic plates, but most likely considering continents. This is also the range of healing time scales in our models, in which reactivation of weak zones can occur. Such tectonic inheritance and its reactivation may be critical in plate tectonics, for example regarding the transition between a mobile, episodic, or stagnant-lid convection, or the cyclicity of plate reorganizations such as for the Wilson cycle. While weakening behavior of grain-size dependent rheologies suggest a fast weakening effect, healing due to grain growth is relatively slow, 
especially considering a two-phase grain size damage which correlates with the lower healing time scales in our models for which reactivation occurs. Given the assemblage of strain over hundreds of millions of years along such suture zones and their preservation of up to $1 \mathrm{Gyr}$, damage weakening might be critical for the cyclicity of the supercontinent cycle.

Rifting processes and the breakup of supercontinents (e.g. Dewey, 1977; Buiter \& Torsvik, 2014) are facilitated by inherited tectonic structures, as it has been observed in Earth's history. Audet \& Brügmann (2011) showed, for example, by an analysis of the effective lithospheric elastic thickness, that the lithospheric strength is controlled by tectonic inheritance and that, during the supercontinent cycle, strain is concentrated along those inherited weak zones. Although we do not consider any continents within our models, strain would be persevered longer in the continental lithosphere, thanks to its density resisting subduction, and this allows for increased longevity of continental weak zones. The formation of such weak zones along passive continental margins and their assemblage during continental reassembling provides a mechanism for continental tectonic inheritance. While the presence of continents influences surface plate motions (e.g. Zhong, 2001; Rolf \& Tackley, 2011; Rolf et al. 2017), strain-dependent weakening would have a clear effect on the breakup and assemblage of continents as well as the plate boundary formation along oceanic-continental boundaries in global convection models. The breakup of continents is also partly controlled by the convecting interior and the location of hotspots (e.g. Hill and Campbell, 1992; Gaina et al., 2007). In our 2-D models, we only assume internal heating, so far, and thus active upwellings are not present in our model. Thus, reactivation is only affected by the lateral drag of the mantle acting on the base of the lithosphere, which has some control on the reactivation of weak zones. However, the question whether reactivation of weak zones or hotspots are the dominant feature remains debated and needs to be explored further using a mixed heated thermal convection. 
As we only consider 2-D thermal convection in the lateral-depth space, any conclusions on the partitioning of surface motion between poloidal and toroidal velocity components as well as the formation of strike-slip plate boundaries are not possible. While toroidal flow is present in 3-D convection models due to temperature-dependent viscosity, it could be enhanced due to the localization and damage weakening effect along plate boundaries. Moreover, the formation of tectonic inheritance and the ability to preserve damage over a geologically long time (up to $1 \mathrm{Gyr}$ ) enhances horizontal variation in the plate viscosity (like a temperature-dependent viscosity), which could further increase toroidal motion at the surface. The presence of tectonic inheritance and more ubiquitous lithospheric inhomogeneities enables the formation of strikeslip or transform plate boundaries (e.g. Zhong \& Gurnis, 1996; Zhong et al., 1998; Bercovici \& Ricard, 2014). Application of a strain-dependent damage weakening rheology in global 3-D spherical convection models might lead to similar surface dynamics and will be investigated in a next step.

In our models, we mainly consider convection for Rayleigh numbers, that are lower than Rayleigh numbers typically assumed for Earth like convection $\left(R a \sim 10^{7}\right)$. While the choice of our Rayleigh number is partially based on numerical limitations, our models show the main characteristics of the mechanics of a straindependent damage-weakening rheology on convection patterns and its time-dependency. The Rayleigh number, however, defines the average velocity for convection and thus the average strain rate and strain accumulation in the lithosphere. Increasing $R a$ would lead to a faster strain accumulation and accordingly a faster strain-dependent weakening. While the healing time scale $B$ should not significantly change with $R a$ (to assure a preservation of strain up to $\sim 1 \mathrm{Gyr}$ ), the weakening time scale (related to the critical strain $\gamma_{c r}$ ) would decrease, which may provide a better match of the strain-dependent damage weakening with parameters inferred from two-phase grain size damage. 


\section{Conclusion}

Simple 2-D convection models including a parameterized strain-dependent weakening rheology allow exploring the time-dependence of plate boundary formation and its effect on convection patterns. Straindependent weakening and, moreover, the ability to preserve inherited lithospheric weak zones over a long geological period, allow the self-consistent formation, preservation, and reactivation of tectonic inheritance. Weak zones can be reactivated in different manners, mainly, as initiation of an intraplate subduction, a ridge adjacent induced subduction, and also as formation of a new spreading centers.

The enhanced weakening along active plate boundaries and the reactivation of weak zones also affects the time dependence of plate reorganization and plate boundary formation and the stability of a plate-like convection. The accumulated damage along spreading centers as well as the inherited weak zones from remnants of subduction zones reduce the average yield stress in the shallow part of the lithosphere. The weakening of the lithosphere and along the plate boundaries results in an increased relative mobility for a plate-like convection (i.e. the period convection is in a mobile-lid) and allows convection in a mobile stage for higher, undeformed lithospheric yield stresses.

While relative mobility is increased due to the tectonic inheritance and reduced average lithospheric yield stress, strain-dependent damage weakening also results in a more pronounced strain localization along active convergent plate boundaries. If weakening along convergent boundaries is efficient enough, the longevity and stability of subduction zones is increased. Thus, strain-dependent weakening results in a shift of the mobile-stagnant lid transition to higher effective yield stresses, if the weak zones fully penetrate the lithosphere and are relatively weakened by at least $20 \%$. 


\section{Acknowledgment}

We like to thank Nicolas Coltice for constructive discussion and two anonymous reviewers whose comments helped to improve the manuscript. The authors acknowledge the Texas Advanced Computing Center (TACC) at The University of Texas at Austin for providing HPC resources that have contributed to the research results reported within this paper. URL: http://www.tacc.utexas.edu. The research project was designed jointly. Computations were performed by Lukas Fuchs who also led writing of the manuscript, to which Thorsten W. Becker contributed. Both authors contributed to the analysis.

\section{References}

Audet, P., \& Bürgmann, R., 2011. Dominant role of tectonic inheritance in supercontinent cycles, Nature Geoscience, 4(3), 184-187.

Austin, N. J., \& Evans, B., 2007. Paleowattmeters: A scaling relation for dynamically recrystallized grain size, Geology, 35(4), 343-346.

Baes, M., Govers, R., \& Wortel, R., 2011. Subduction initiation along the inherited weakness zone at the edge of a slab: Insights from numerical models, Geophysical Journal International, 184(3), 991-1008.

Becker, T. W., \& Faccenna, C., 2009. A review of the role of subduction dynamics for regional and global plate motions, In Subduction Zone Geodynamics (pp. 3-34), Springer, Berlin, Heidelberg.

Bercovici, D., 1993. A simple model of plate generation from mantle flow, Geophysical Journal International, 114(3), 635-650.

Bercovici, D., 1995. On the purpose of toroidal motion in a convecting mantle, Geophysical Research Letters, 22(23), 3107-3110.

Bercovici, D., 2003. The generation of plate tectonics from mantle convection, Earth and Planetary Science Letters 205: 107-121.

Bercovici, D., \& Ricard, Y., 2005. Tectonic plate generation and two-phase damage: Void growth versus grain size reduction, Journal of Geophysical Research: Solid Earth, 110(B3). 
Bercovici, D., \& Ricard, Y., 2012. Mechanisms for the generation of plate tectonics by two-phase graindamage and pinning, Physics of the Earth and Planetary Interiors, 202, 27-55.

Bercovici, D., \& Ricard, Y., 2013. Generation of plate tectonics with two-phase grain-damage and pinning: Source-sink model and toroidal flow, Earth and Planetary Science Letters, 365, 275-288.

Bercovici, D., \& Ricard, Y., 2014. Plate tectonics, damage and inheritance, Nature, 508(7497), 513.

Bercovici, D., \& Ricard, Y., 2016. Grain-damage hysteresis and plate tectonic states, Physics of the Earth and Planetary Interiors, 253, 31-47.

Bercovici, D., Tackley, P., \& Ricard, Y., 2015. 7.07-the generation of plate tectonics from mantle dynamics, Treatise on Geophysics, Elsevier, Oxford, 271-318.

Billen, M. I., \& Hirth, G., 2007. Rheologic controls on slab dynamics. Geochemistry, Geophysics, Geosystems, 8(8).

Braun, J., Chéry, J., Poliakov, A., Mainprice, D., Vauchez, A., Tomassi, A., \& Daignières, M., 1999. A simple parameterization of strain localization in the ductile regime due to grain size reduction: A case study for olivine, Journal of Geophysical Research: Solid Earth, 104(B11), 25167-25181.

Buiter, S. J., \& Torsvik, T. H., 2014. A review of Wilson Cycle plate margins: A role for mantle plumes in continental break-up along sutures?, Gondwana Research, 26(2), 627-653.

Butler, R. W., Tavarnelli, E., \& Grasso, M., 2006. Structural inheritance in mountain belts: an AlpineApennine perspective, Journal of Structural Geology, 28(11), 1893-1908.

Coltice, N., Gérault, M., \& Ulvrová, M., 2017. A mantle convection perspective on global tectonics, Earthscience reviews, 165, 120-150.

Dannberg, J., Eilon, Z., Faul, U., Gassmöller, R., Moulik, P., \& Myhill, R., 2017. The importance of grain size to mantle dynamics and seismological observations, Geochemistry, Geophysics, Geosystems, 18(8), 3034-3061.

Dewey, J. F., 1977. Suture zone complexities: a review, Tectonophysics, 40(1-2), 53-67. 
Enns, A., Becker, T. W., \& Schmeling, H., 2005. The dynamics of subduction and trench migration for viscosity stratification, Geophysical Journal International, 160(2), 761-775.

Foley, B. J., \& Becker, T. W., 2009. Generation of plate-like behavior and mantle heterogeneity from a spherical, viscoplastic convection model, Geochemistry, Geophysics, Geosystems, 10, Q08001.

Gaina, C., Müller, R. D., Brown, B., Ishihara, T., \& Ivanov, S., 2007. Breakup and early seafloor spreading between India and Antarctica. Geophysical Journal International, 170(1), 151-169.

Gerya, T., 2010. Dynamical instability produces transform faults at mid-ocean ridges. Science, 329(5995), 1047-1050.

Gueydan, F., \& Précigout, J., 2014. Modes of continental rifting as a function of ductile strain localization in the lithospheric mantle, Tectonophysics, 612, 18-25.

Gueydan, F., Précigout, J., \& Montési, L. G., 2014. Strain weakening enables continental plate tectonics, Tectonophysics, 631, 189-196.

Gurnis, M., Zhong, S., \& Toth, J., 2000. On the competing roles of fault reactivation and brittle failure in generating plate tectonics from mantle convection. M.A. Richards, R.G. Gordon, R.D. van der Hilst (Eds.), The History and Dynamics of Global Plate Motions, American Geophysical Union (2000), pp. 73-94.

Hall, C. E., \& Parmentier, E. M., 2003. Influence of grain size evolution on convective instability, Geochemistry, Geophysics, Geosystems, 4(3).

Hansen, L. N., Zimmerman, M. E., \& Kohlstedt, D. L., 2012. The influence of microstructure on deformation of olivine in the grain-boundary sliding regime, Journal of Geophysical Research: Solid Earth, 117(B9).

Hill, R. I., Campbell, I. H., Davies, G. F., \& Griffiths, R. W., 1992. Mantle plumes and continental tectonics. Science, 256(5054), 186-193.

Holt, A. F., Becker, T. W., \& Buffett, B. A., 2015. Trench migration and overriding plate stress in dynamic subduction models, Geophysical Journal International, 201(1), 172-192. 
Huismans, R. S., \& Beaumont, C., 2003. Symmetric and asymmetric lithospheric extension: Relative effects of frictional-plastic and viscous strain softening, Journal of Geophysical Research: Solid Earth, 108(B10).

Huismans, R. S., Buiter, S. J., \& Beaumont, C., 2005. Effect of plastic-viscous layering and strain softening on mode selection during lithospheric extension, Journal of Geophysical Research: Solid Earth, 110(B2).

Karato, S. I., 1989. Grain growth kinetics in olivine aggregates, Tectonophysics, 168(4), 255-273.

Karato, S. I., Toriumi, M., \& Fujii, T., 1980. Dynamic recrystallization of olivine single crystals during high-temperature creep, Geophysical Research Letters, 7(9), 649-652.

Karato, S. I., Paterson, M. S., \& FitzGerald, J. D., 1986. Rheology of synthetic olivine aggregates: influence of grain size and water, Journal of Geophysical Research: Solid Earth, 91(B8), 8151-8176.

Kohlstedt, D. L., Evans, B., \& Mackwell, S. J., 1995. Strength of the lithosphere: Constraints imposed by laboratory experiments, Journal of Geophysical Research: Solid Earth, 100(B9), 17587-17602.

Landuyt, W. Bercovici, D. \& Ricard, Y., 2008. Plate generation and two-phase damage theory in a model of mantle convection, Geophysical Journal International, 174, 1065-1080.

Landuyt, W., \& Bercovici, D., 2009. Formation and structure of lithospheric shear zones with damage. Physics of the Earth and Planetary Interiors, 175(3-4), 115-126.

Lavier, L. L., Buck, W. R. \& Poliakov, A. N. B., 2000. Factors controlling normal fault offset in an ideal brittle layer, Journal of Geophysical Research: Solid Earth, 105(B10), 23431-23442.

Mazzotti, S., \& Gueydan, F., 2017. Control of tectonic inheritance on continental intraplate strain rate and seismicity, Tectonophysics, in press.

Montési, L. G., \& Hirth, G., 2003. Grain size evolution and the rheology of ductile shear zones: from laboratory experiments to postseismic creep, Earth and Planetary Science Letters, 211(1-2), 97-110.

Montési, L. G., 2013. Fabric development as the key for forming ductile shear zones and enabling plate tectonics, Journal of Structural Geology, 50, 254-266. 
Moresi, L., \& Gurnis, M., 1996. Constraints on the lateral strength of slabs from three-dimensional dynamic flow models, Earth and Planetary Science Letters, 138(1-4), 15-28.

Mouthereau, F., Watts, A. B., \& Burov, E., 2013. Structure of orogenic belts controlled by lithosphere age, Nature geoscience, 6(9), 785.

O’Connell, R.J., Gable, C.W. \& Hager, B.H., 1991. Toroidal-poloidal partitioning of lithospheric plate motions, In Glacial Isostasy, Sea-Level and Mantle Rheology (pp. 535-551). Springer, Dordrecht.

Ogawa, M., 2003. Plate-like regime of a numerically modeled thermal convection in a fluid with temperature-, pressure-, and stress-history-dependent viscosity, Journal of Geophysical Research: Solid Earth, 108(B2).

Précigout, J., \& Almqvist, B. S., 2014. The Ronda peridotite (Spain): A natural template for seismic anisotropy in subduction wedges. Geophysical Research Letters, 41(24), 8752-8758.

Ricard, Y., \& Bercovici, D., 2009. A continuum theory of grain size evolution and damage, Journal of Geophysical Research: Solid Earth, 114(B1).

Richards, M. A., Yang, W. S., Baumgardner, J. R., \& Bunge, H. P., 2001. Role of a low-viscosity zone in stabilizing plate tectonics: Implications for comparative terrestrial planetology, Geochemistry, Geophysics, Geosystems, 2(8).

Rolf, T., \& Tackley, P. J., 2011. Focusing of stress by continents in 3D spherical mantle convection with self-consistent plate tectonics, Geophysical Research Letters, 38(18).

Rolf, T., Capitanio, F. A., \& Tackley, P. J., 2017. Constraints on mantle viscosity structure from continental drift histories in spherical mantle convection models, Tectonophysics, in press.

Rozel, A., Ricard, Y., \& Bercovici, D., 2011. A thermodynamically self-consistent damage equation for grain size evolution during dynamic recrystallization, Geophysical Journal International, 184(2), 719728.

Schubert, G., \& Turcotte, D. L., 1972. One-dimensional model of shallow-mantle convection, Journal of Geophysical Research, 77(5), 945-951. 
Solomatov, V. S., 1995. Scaling of temperature-and stress-dependent viscosity convection, Physics of Fluids, 7(2), 266-274.

Solomatov, V.S., 2001. Grain size-dependent viscosity convection and the thermal evolution of the Earth, Earth and Planetary Science Letters, Volume 191, Issues 3-4, 2001, Pages 203-212.

Solomatov, V. S., \& Moresi, L. N., 1996. Stagnant lid convection on Venus, Journal of Geophysical Research: Planets, 101(E2), 4737-4753.

Sykes, L. R., 1978. Intraplate seismicity, reactivation of preexisting zones of weakness, alkaline magmatism, and other tectonism postdating continental fragmentation, Reviews of Geophysics, 16(4), 621-688.

Tackley, P. J., 1998. Self-consistent generation of tectonic plates in three-dimensional mantle convection, Earth and Planetary Science Letters, 157(1-2), 9-22.

Tackley, P. J., 2000a. Self-consistent generation of tectonic plates in time-dependent, three-dimensional mantle convection simulations: 1. Pseudoplastic yielding, Geochemistry, Geophysics, Geosystems, 1(8).

Tackley, P. J., 2000b. Self-consistent generation of tectonic plates in time-dependent, three-dimensional mantle convection simulations: 2. Strain weakening and asthenosphere, Geochemistry, Geophysics, Geosystems, 1(8), 1026.

Thielmann, M., \& Kaus, B. J., 2012. Shear heating induced lithospheric-scale localization: Does it result in subduction?, Earth and Planetary Science Letters, 359, 1-13.

Tommasi, A., Knoll, M., Vauchez, A., Signorelli, J.W., Thoraval, C. \& Loge, R., 2009. Structural reactivation in plate tectonics controlled by olivine crystal anisotropy, Nature Geosciences, v. 2, p. 423427.

Van Heck, H. J., \& Tackley, P. J., 2008. Planforms of self-consistently generated plates in 3D spherical geometry, Geophysical Research Letters, 35(19).

Weinstein, S. A., \& Olson, P. L., 1992. Thermal convection with non-Newtonian plates, Geophysical Journal International, 111(3), 515-530. 
Wilson, J. T., 1966. Did the Atlantic close and then re-open?, Nature, 211, 676-681.

Zhong, S., 2001. Role of ocean-continent contrast and continental keels on plate motion, net rotation of lithosphere, and the geoid, Journal of Geophysical Research: Solid Earth, 106(B1), 703-712.

Zhong, S., 2006. Constraints on thermochemical convection of the mantle from plume heat flux, plume excess temperature, and upper mantle temperature, Journal of Geophysical Research: Solid Earth, 111(B4).

Zhong, S., \& Gurnis, M., 1995a. Mantle convection with plates and mobile, faulted plate margins, Science, 267(5199), 838-843.

Zhong, S., \& Gurnis, M., 1995b. Towards a realistic simulation of plate margins in mantle convection, Geophysical Research Letters, 22(8), 981-984.

Zhong, S. \& Gurnis, M., 1996. Interaction of weak faults and non-Newtonian rheology produces plate tectonics in a 3D model of mantle flow, Nature, 383, 245-247.

Zhong, S., Gurnis, M. \& Moresi, L., 1998. Role of faults, nonlinear rheology, and viscosity structure in generating plates from instantaneous mantle flow models, Journal of Geophysical Research, 103, 15255-15268.

Zhong, S., Zuber, M. T., Moresi, L., \& Gurnis, M., 2000. Role of temperature-dependent viscosity and surface plates in spherical shell models of mantle convection, Journal of Geophysical Research: Solid Earth, 105(B5), 11063-11082. 
Figures and Tables

Table 1: Model and Scaling Parameters

\begin{tabular}{|c|c|c|c|c|}
\hline & Symbol & Scaling & Value & Unit \\
\hline Length & $(x, z)$ & $D$ & $2871 \cdot 10^{3}$ & $\mathrm{~m}$ \\
\hline Time & $t_{O T}$ & $\frac{2 D}{v_{\text {char }}}$ & 114.84 & $\mathrm{Ma}$ \\
\hline Viscosity & $\eta$ & $\eta_{r e f}$ & $10^{23}$ & Pas \\
\hline Scaling stress & $\sigma_{y, s c}$ & $\frac{\eta_{r e f} \kappa}{D^{2}}$ & - & $\mathrm{MPa}$ \\
\hline Stress & $\sigma_{y}$ & $\sigma_{y . s c}$ & {$\left[10^{3}-2 \cdot 10^{4}\right]$} & - \\
\hline Rayleigh number & $R a$ & - & {$\left[5 \cdot 10^{4}, 10^{5}, 5 \cdot 10^{5}\right]$} & - \\
\hline Healing time scale & $B$ & $t_{O T}$ & [0.36-362.04] & $1 /$ number of OT \\
\hline Critical strain & $\gamma_{c r}$ & - & [0.94-18] & - \\
\hline Thermal diffusivity & $\kappa$ & - & $10^{-6}$ & $\mathrm{~m}^{2} / \mathrm{s}$ \\
\hline $\begin{array}{c}\text { Temperature } \\
\text { difference }\end{array}$ & $\Delta T$ & - & 1300 & $\mathrm{~K}$ \\
\hline Density & $\rho$ & - & 3300 & $\mathrm{~kg} / \mathrm{m}^{3}$ \\
\hline $\begin{array}{l}\text { Gravitational } \\
\text { acceleration }\end{array}$ & $g$ & - & 9.81 & $\mathrm{~m} / \mathrm{s}^{2}$ \\
\hline Thermal expansivity & $\alpha$ & - & $1 e-5$ & $\mathrm{~K}^{-1}$ \\
\hline
\end{tabular}




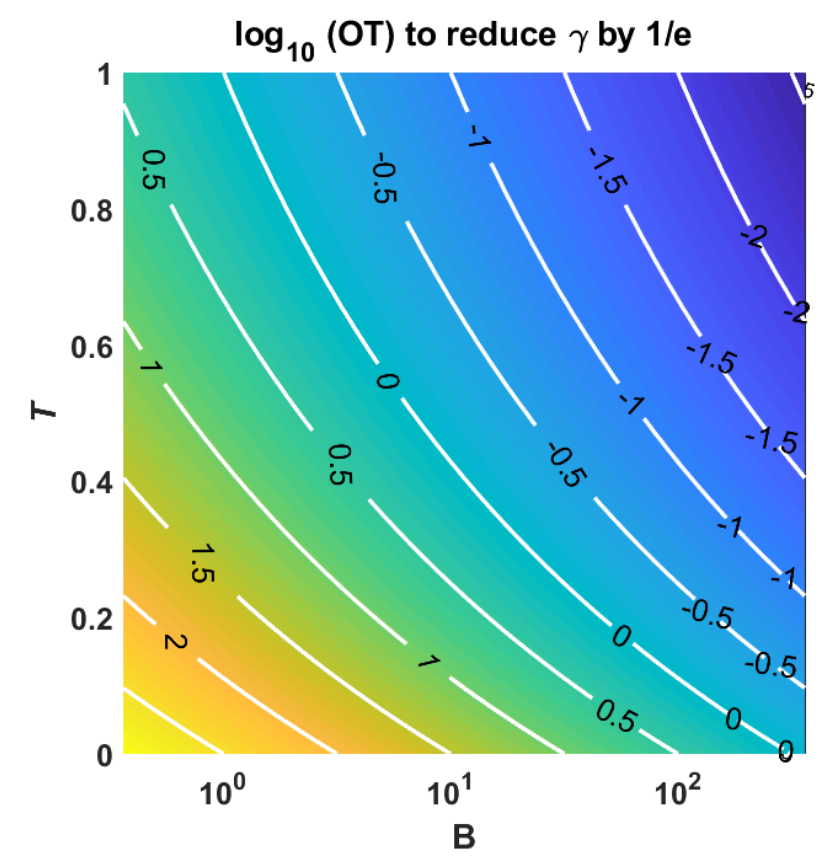

Figure 1: Strain reduction time (background color and solid contour lines) to reduce $\gamma$ by a factor of 1/e for different temperature and healing time scales. The time is calculated using eq. (8) assuming no deformation is active, i.e. the strain-rate is zero. If $T=1$, the total healing rate $H$ is equal to the healing time scale $B$ and thus the reduction time is $1 / B$. With decreasing $T$, the healing rate decreases as well and the reduction time increases to $c / B$, where $c$ is a temperature dependent constant (see eq. (9) for more detail; see online version for color) 

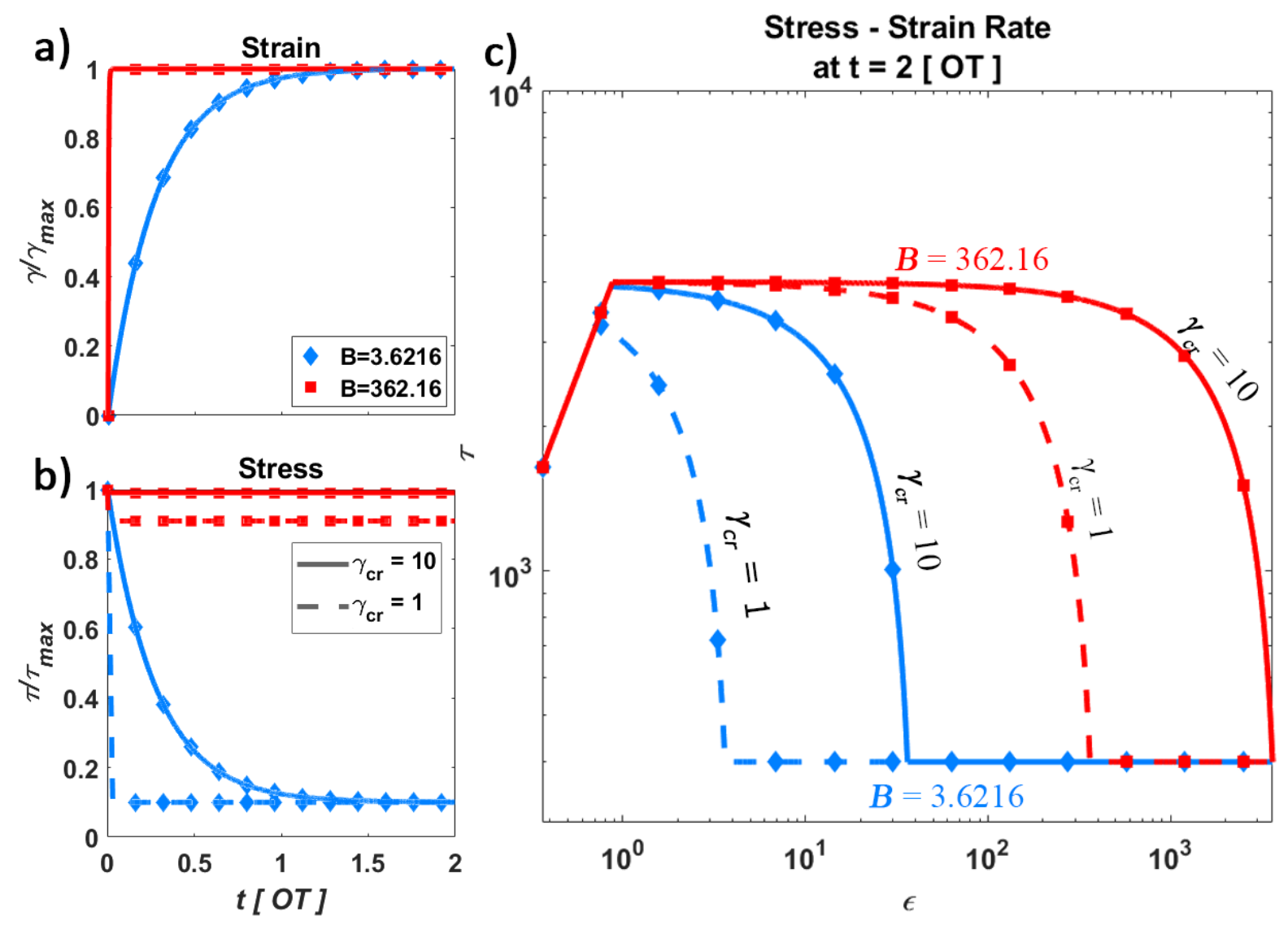

Figure 2: Deformation map for damage weakening rheology assuming a constant strain rate over a duration of 2 OT. a) Evolution of strain (scaled by $\gamma_{\max }$ ) for different healing time scales, $B$. b) Evolution of stress (scaled by $\tau_{\max }$ ) for different healing time scales $B$ (squares/red and diamond/blue) and critical strains $\gamma_{c r}$ (solid and dashed). The "undeformed" yield stress is $4 \cdot 10^{3}$. c) Stress - strain rate relation for a range of $B$ and $\gamma_{c r}$ for constant strain rates $\dot{\epsilon}$ after a total time of $t=2$ OT. A higher $B$ and $\gamma_{c r}$ result in less accumulated strain and weakening, thus the yield stress is reduced only slightly. A stronger weakening due to lower $B$ and $\gamma_{c r}$ results in stronger reduction in yield stress (see online version for color). 

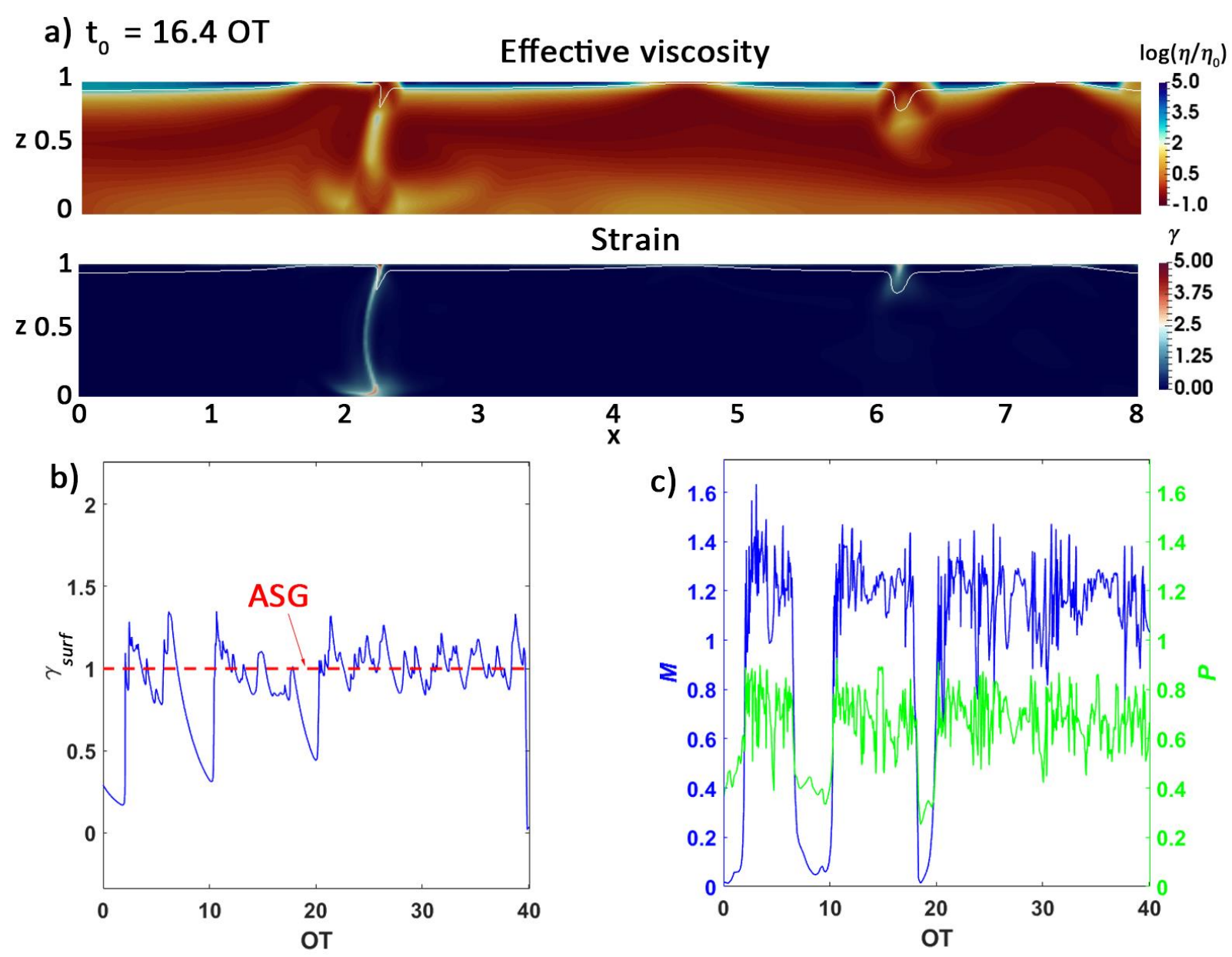

Figure 3: Reference model for pseudo-plastic convection. a) Effective viscosity $\eta$ and strain $\gamma$ at a certain time step showing the correlation between strain and convection dynamics. The white contour line shows the 0.3 iso-temperature contour. b) Surface-averaged strain $\gamma_{\text {surf }}$ (solid line) and time-averaged surfaceaverage strain (ASG - dashed lined). c) Mobility ( $M$ - dark grey, blue) and plateness ( $P$ - light grey, green) over an arbitrary period of 40 overturn times (OT) during the entire model period (see online version for color). 

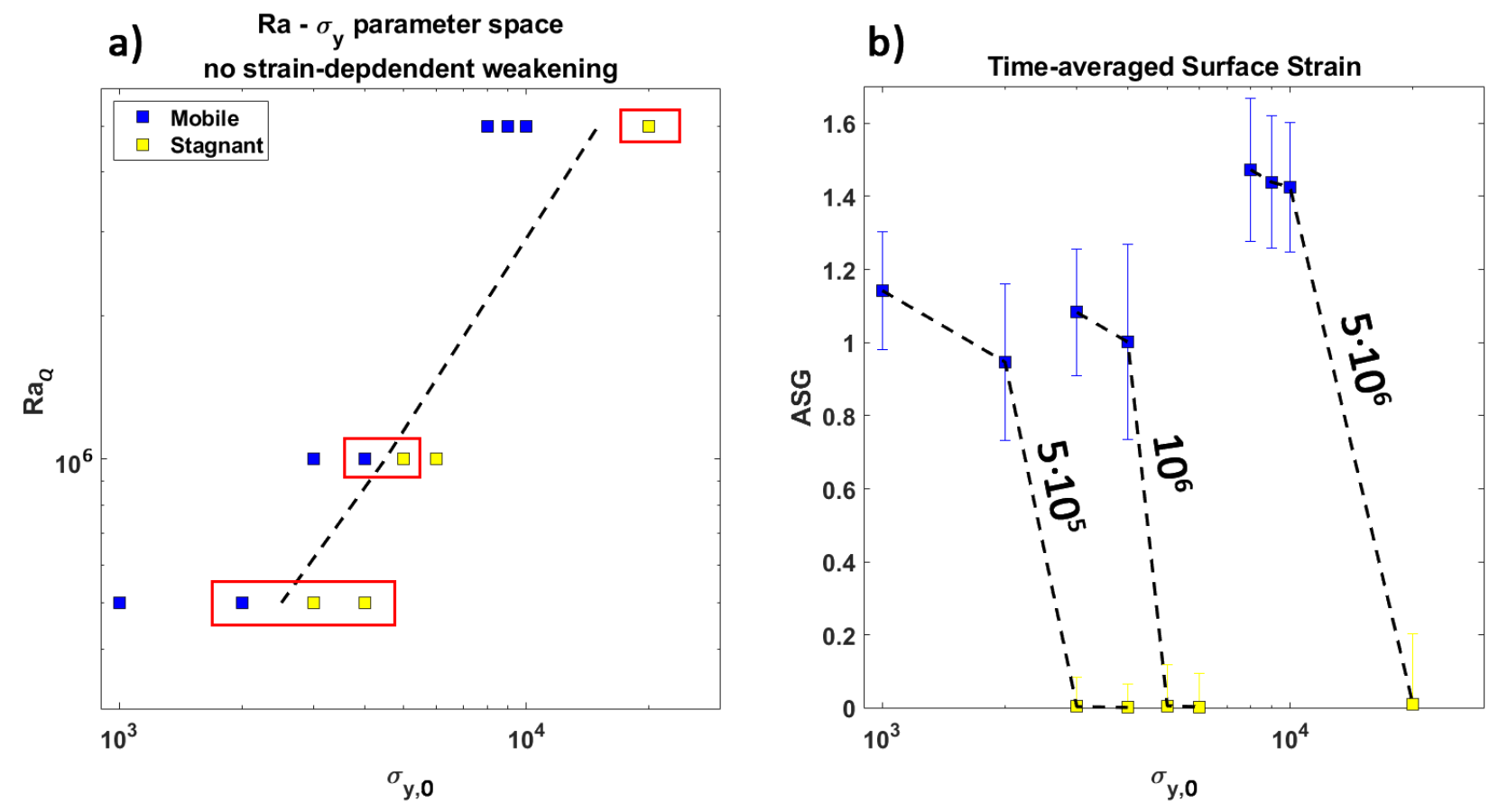

Figure 4: $R a_{Q}-\sigma_{y, 0}$ parameter space (a) and time-averaged surface-average strain (ASG) and standard deviations due to oscillating average surface strain $\gamma_{\text {surf }}(\mathrm{b}, \mathrm{ASG})$ for models without strain-dependent weakening (SDW). The dashed line in a) indicates the transition between mobile and stagnant-lid convection. The red rectangles indicate the 'undeformed' $R a_{Q^{-}} \sigma_{y, 0}$ parameter combinations for model runs including SDW (see Fig. 11). The dashed lines in b) are for ASG values of the same Ra. 


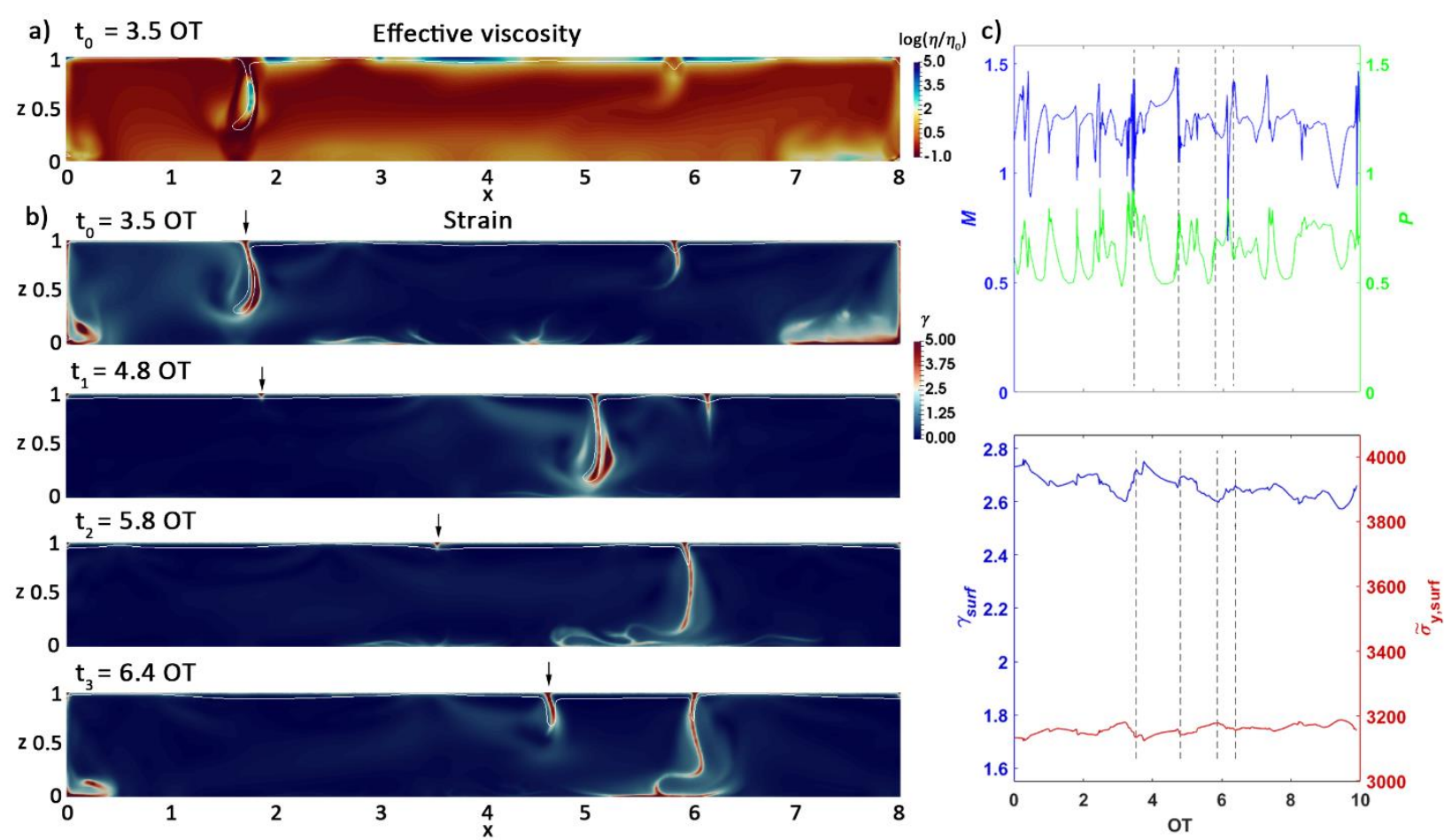

Figure 5: Subduction re-initiation for a model with low healing time scale $B(0.3622)$ and high critical strain $\gamma_{c r}(10)$. a) Effective viscosity at 3.5 OT. The white line shows the 0.3 iso-temperature contour. b) Evolution of the strain field. The black arrows indicate the re-initiation of subduction at an inherited selfconsistently evolving weak zone in the lithosphere. c) Top: Time series of mobility ( $M$ - dark grey, blue) and plateness $\left(P\right.$ - light grey, green). Bottom: Time series of surface-averaged strain $\left(\gamma_{\text {surf }}-\right.$ top line $)$ and yield stress $\left(\sigma_{y, \text { surf }}\right.$ - bottom line). The black, vertical dashed lines highlight the time steps for the snap shots in $b$ (see online version for color). 

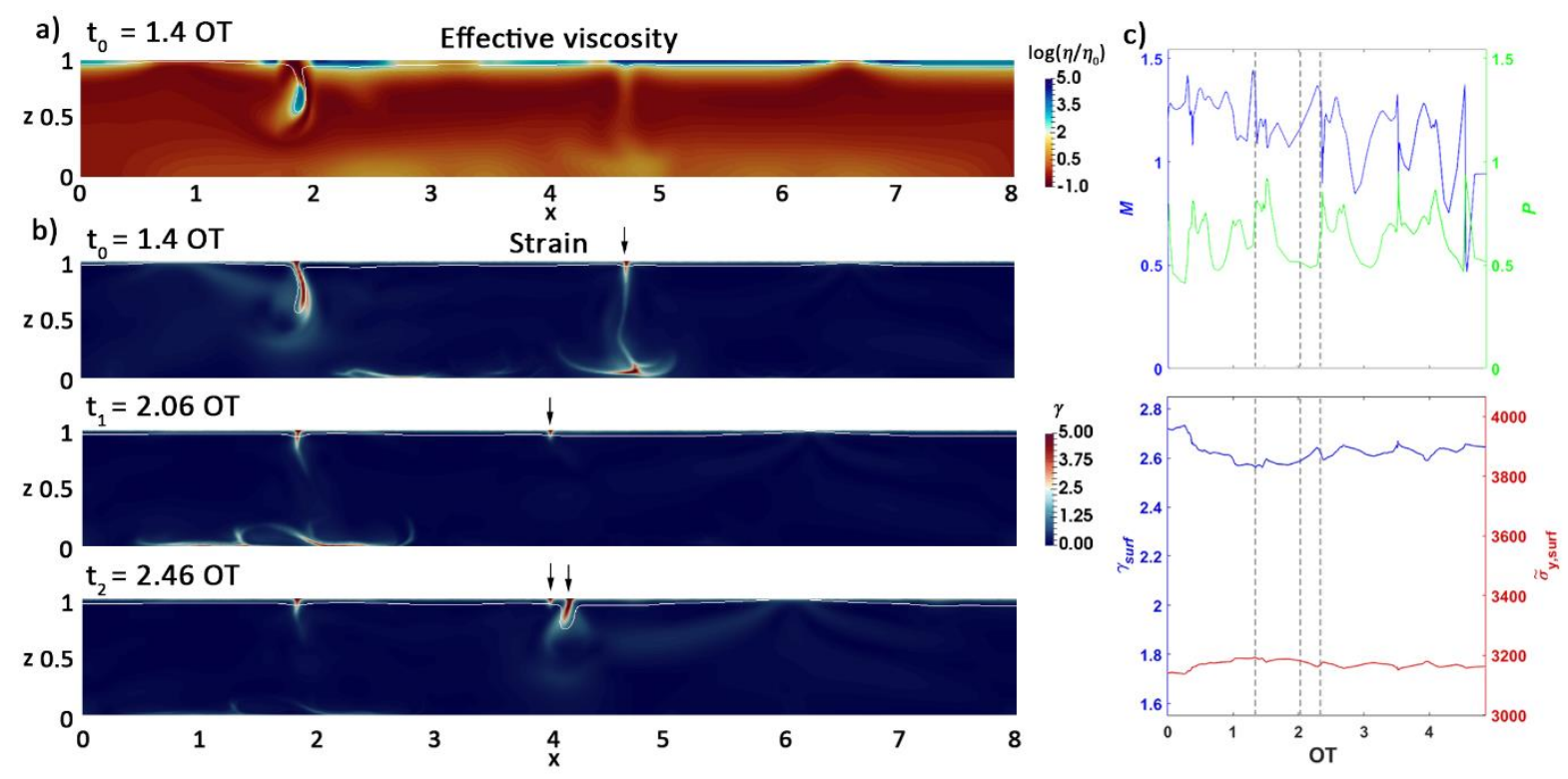

Figure 6: "Failed" subduction initiation for the same model as shown in Figure 3 with a low healing time scale $B(0.3622)$ and high critical strain $\gamma_{c r}(10)$. a and b) Initial effective viscosity $\eta$ and time evolution of the strain $\gamma$. c) Time series of (at the top) mobility ( $M$ - dark grey, blue), plateness ( $P$ - light grey, green), and (at the bottom) surface-averaged strain ( $\gamma_{\text {surf }}-$ top line) and surface yield stress ( $\sigma_{y, s u r f}$ - bottom line; see online version for color). 

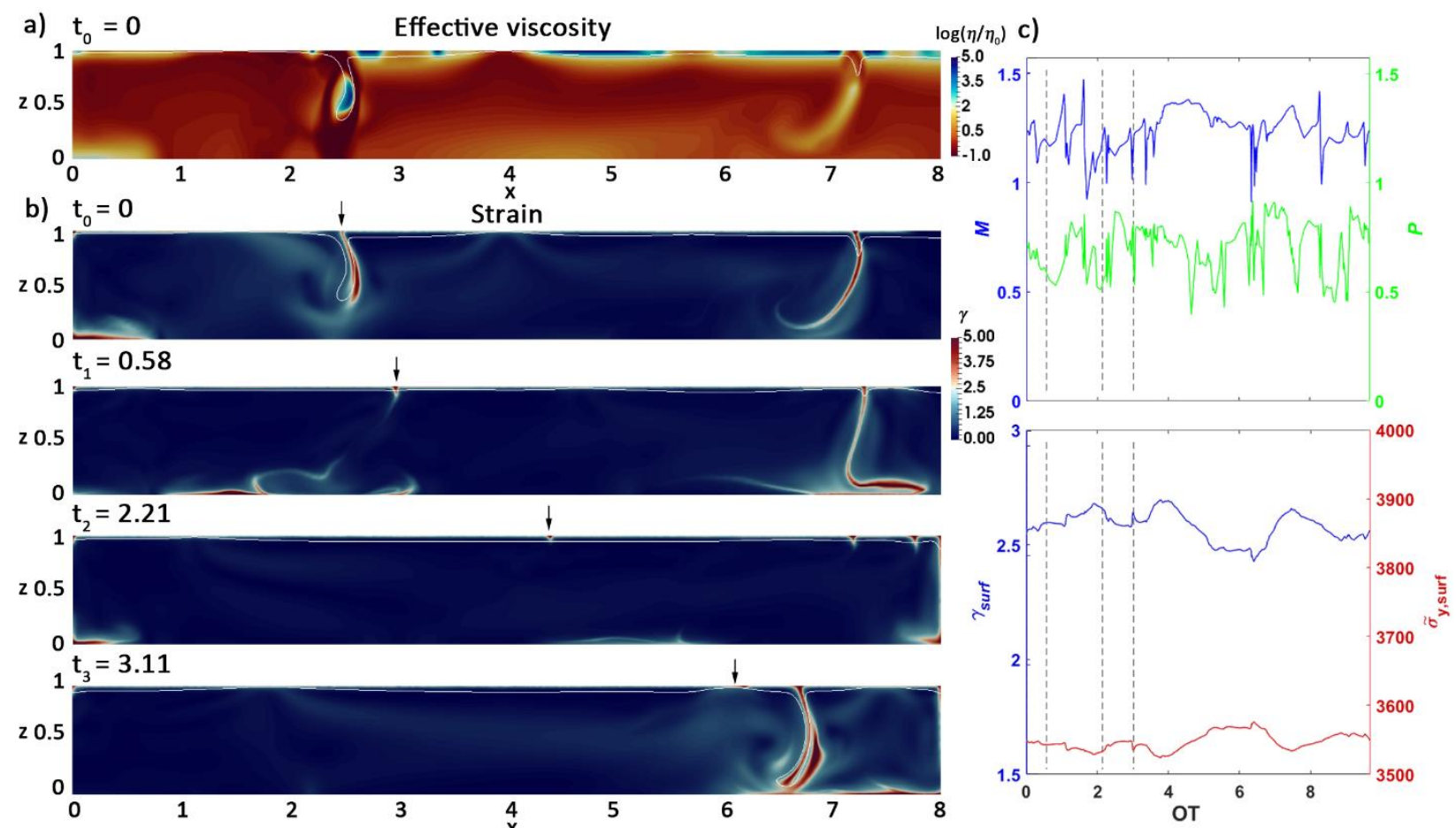

Figure 7: Spreading center initiation for a model with a high critical strain $\gamma_{c r}$ (18) and low healing time scale $B(0.3622)$. a and b) Initial effective viscosity $\eta$ and time evolution of the strain $\gamma$.c) Top: Time series of the mobility $(M-$ dark grey, blue) and plateness $(P-$ light grey, green). Bottom: Time series of the surface-averaged strain $\left(\gamma_{\text {surf }}-\right.$ top line $)$ and surface yield stress $\left(\sigma_{y, s u r f}\right.$ - bottom line; see online version for color). 

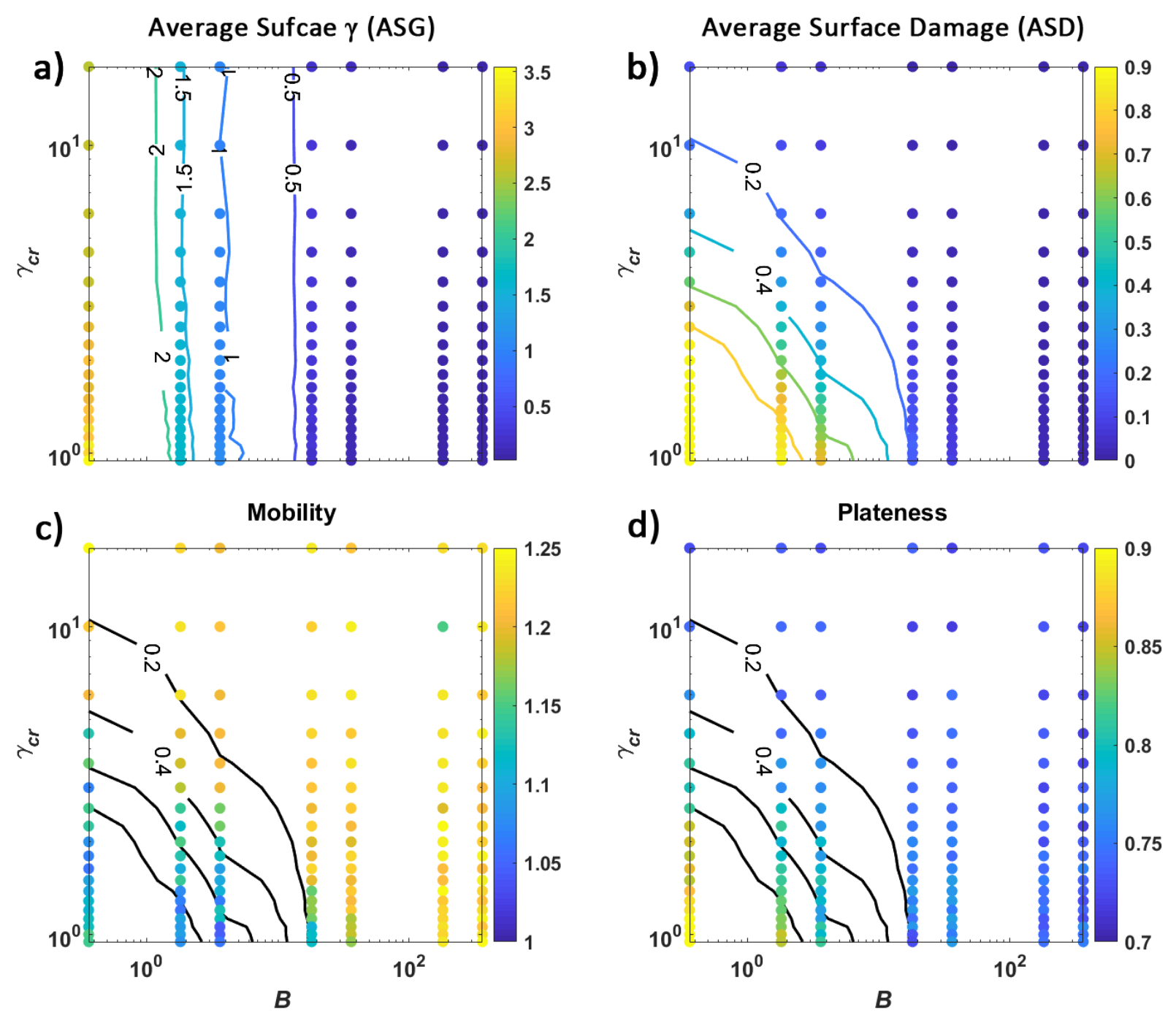

Figure 8: Time-averaged surface-average lithospheric strain (ASG), damage (ASD), mobility and plateness as a function of the healing time scale, $B$, and critical strain, $\gamma_{c r}$. Each of the time-dependent metric for each model are averaged over a period of 40 OT, starting at 10 OT. a) Time-averaged surface-average lithospheric strain ASG. b) Time-averaged surface-average lithospheric damage. c) Mobility. d) Plateness. The contour lines in $\mathrm{c}$ and $\mathrm{d}$ show the ASD as in $\mathrm{b}$ (see online version for color). 

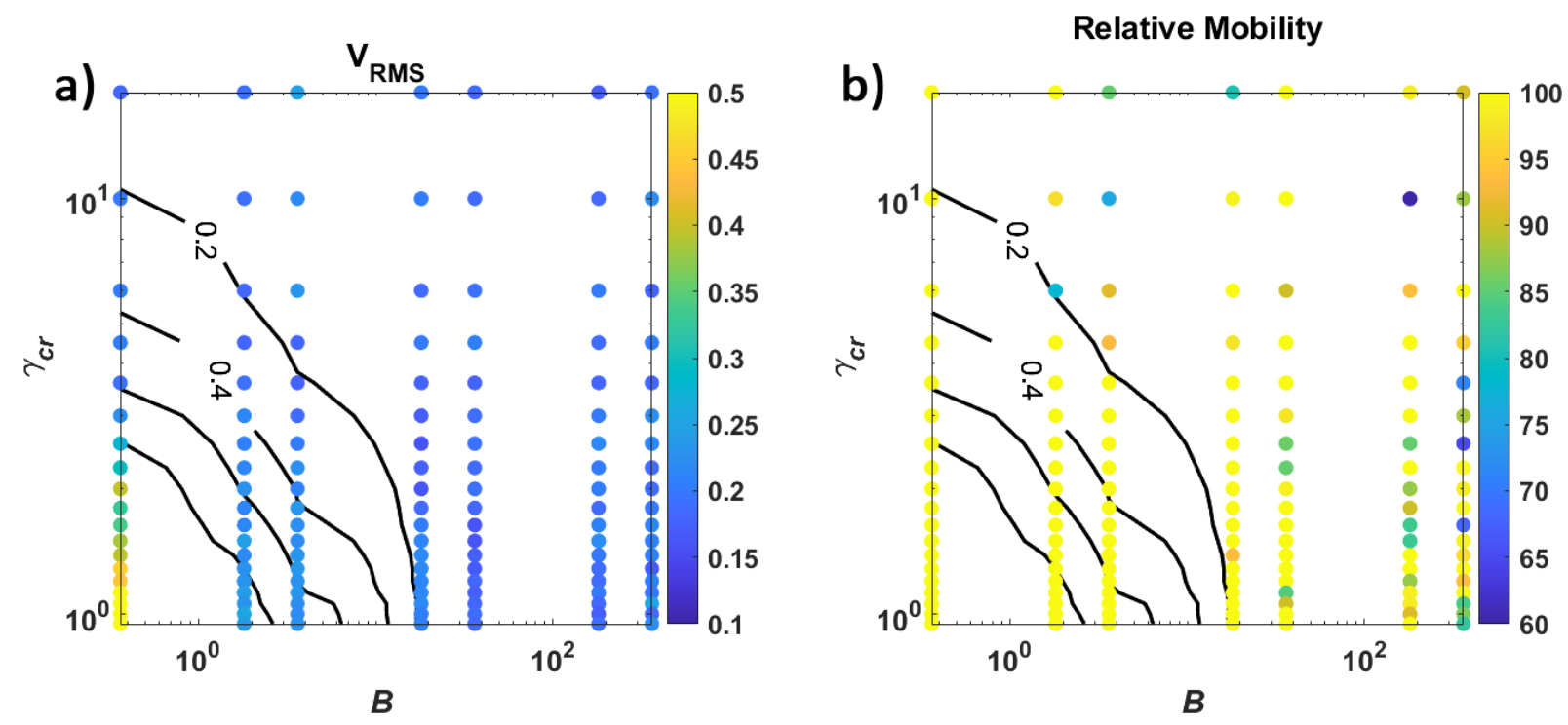

Figure 9: Time-averaged root mean square and relative mobility. The metrics of each model are averaged over a period of 40 OT, starting at 10 OT. The contour lines in a and b show the average lithospheric damage from Figure $8 b$ (see online version for color). 


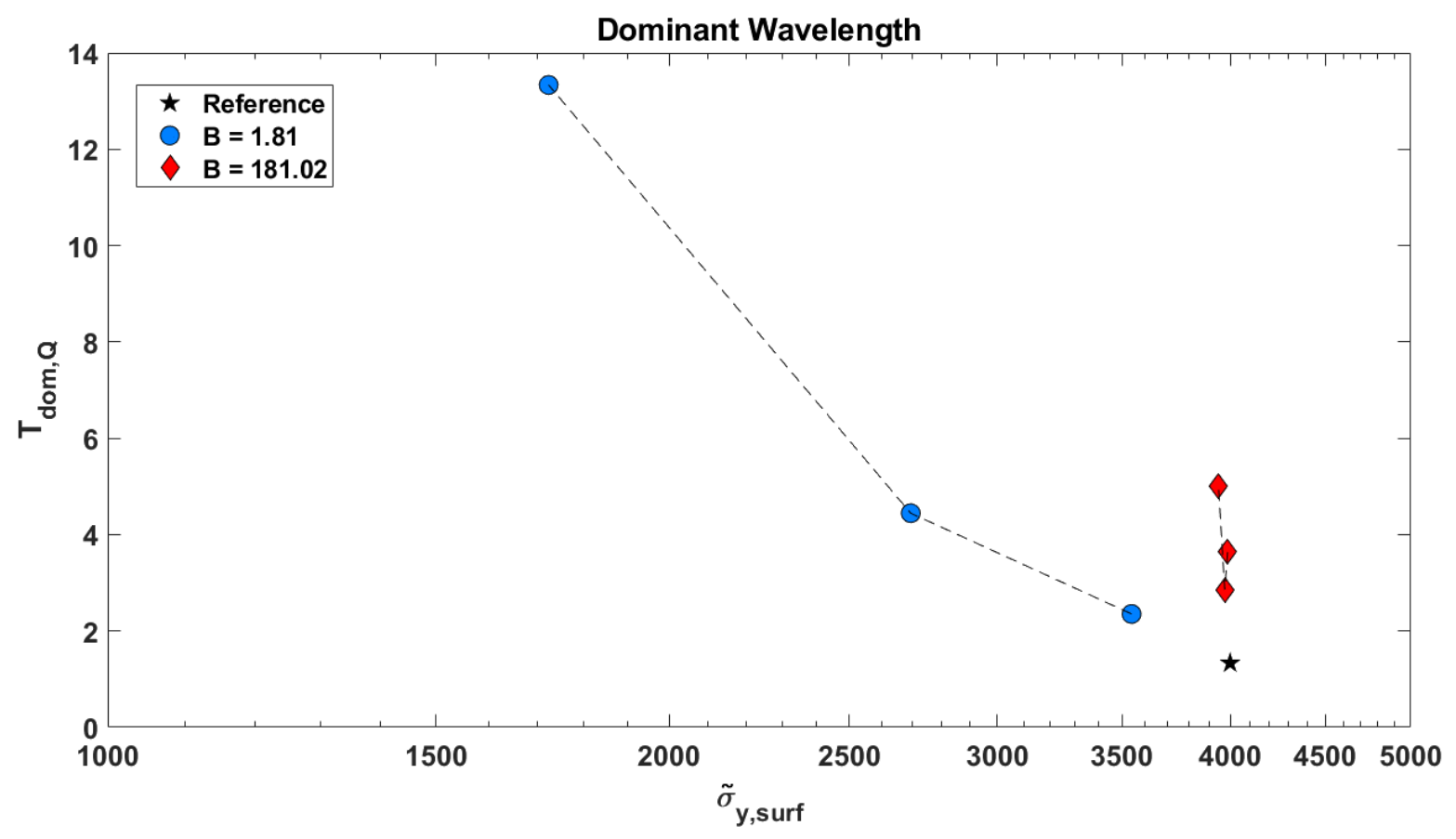

Figure 10: Dominant wavelength of total surface heat flow for models including strain-dependent weakening (SDW) for $R a_{Q}=10^{6}$ and $\sigma_{\mathrm{y}, 0}=4 \cdot 10^{3}$ (Reference model without SDW) and different healing time scales $B$ and critical strains $\gamma_{c r}=10,3.6,2$. In general, SDW shift convection to longer periods and with more dominant weakening (i.e. decreasing $\gamma_{c r}$ ) increases the dominant convection wavelength further (see online version for color). 


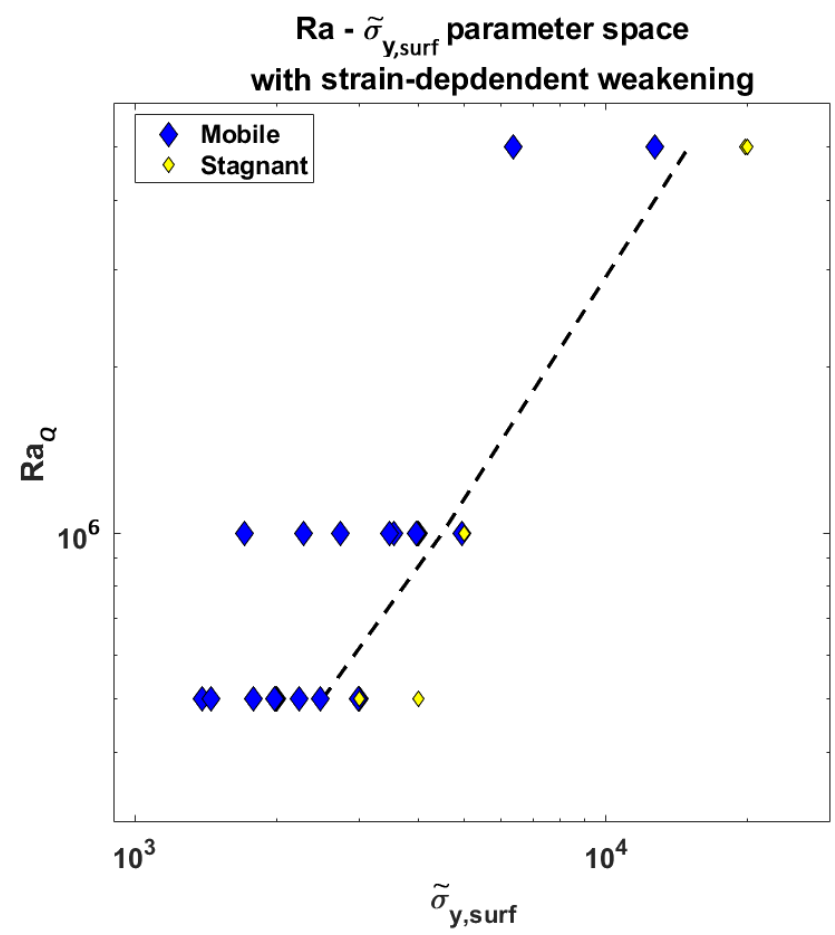

Figure 11: $R a_{Q^{-}} \sigma_{y}$ parameter space for models with strain-dependent weakening (SDW). The black dashed line indicates the transition between mobile (large diamonds) and stagnant-lid (small diamonds) convection for models without SDW. For models including SDW, we chose a model in a mobile stage adjacent to the boundary as initial condition and increased the yield stress step wise, i.e. $R a_{Q}=5 \cdot 10^{5}, \sigma_{y, 0}=2 \cdot 10^{3}, 3 \cdot 10^{3}$, $4 \cdot 10^{3} ; R a_{Q}=10^{6}, \sigma_{y, 0}=4 \cdot 10^{3}, 5 \cdot 10^{3} ; R a_{Q}=5 \cdot 10^{6}, \sigma_{y, 0}=2 \cdot 10^{4}$. For each of those $R a_{Q}-\sigma_{y, O}$ combinations we run six models using a certain parameter combination for $B$ and $\gamma_{c r}\left(B=1.81,181.02 ; \gamma_{c r}=10,3.6,2\right)$. A model is considered stagnant if the mean of the mobility over the full model time is less than 0.1 (see online version for color). 
a)
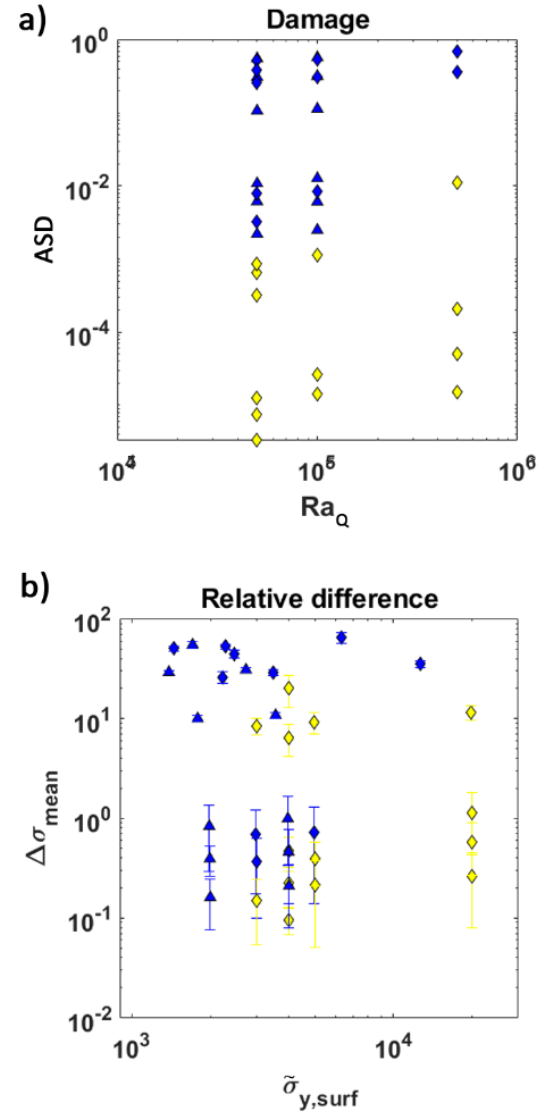
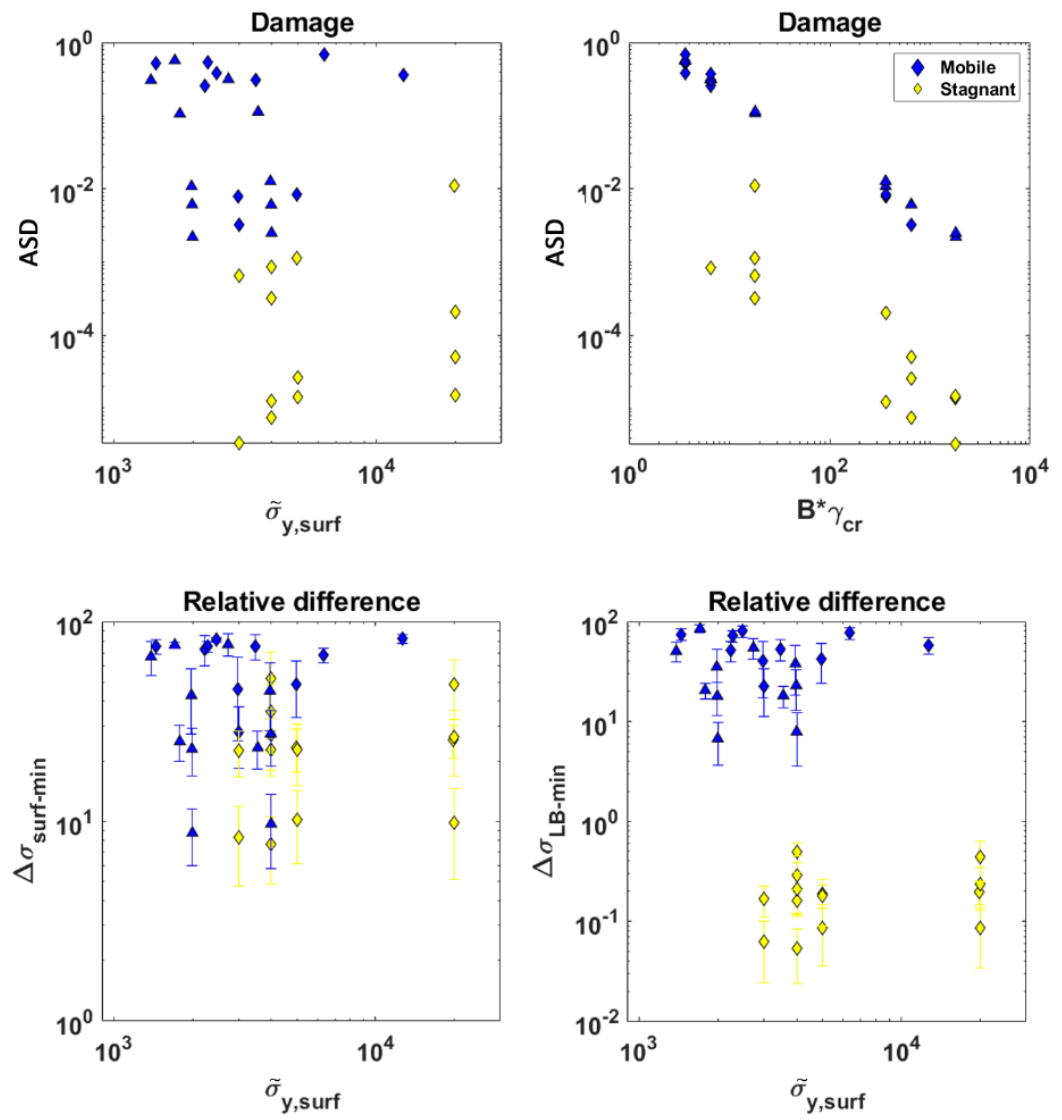

Figure 12: Parameter space for time-averaged surface-average damage $A S D$ (a) and relative difference in stresses (b) relative to different parameters for models including strain-dependent weakening (SDW). The parameters are averaged over the longest mobile-lid period. A model is considered stagnant if the mean of the mobility over the full model time is less than 0.1 . a) Damage relative to $R a_{Q}, \tilde{\sigma}_{y, s u r f}$, and $B \cdot \gamma_{c r}$. The damage is defined by eq. (11). b) Relative difference in stresses relative to the effective surface yield stress $\tilde{\sigma}_{y, \text { surf. }} \Delta \sigma_{\text {mean }}-$ Difference of mean surface and mean lithospheric base (LB) yield stress, $\Delta \sigma_{y, L B-m i n}-$ Difference of mean LB yield stress and minimum LB yield stress, $\Delta \sigma_{\text {surf-min }}-$ Difference between mean surface yield stress and minimum surface yield stress. Triangles and diamonds distinguish between $R a-\sigma_{y, 0}$ parameter combinations in mobile stage and stagnant-lid, respectively, for models without weakening (see online version for color). 